\title{
Selection and Implementation of a Replacement Cutting Tool Selection Application
}

Federal Manufacturing \& Technologies

Gordon Rice

KCP-613-8512

September 2008

Final Report

Approved for public release; distribution is unlimited.

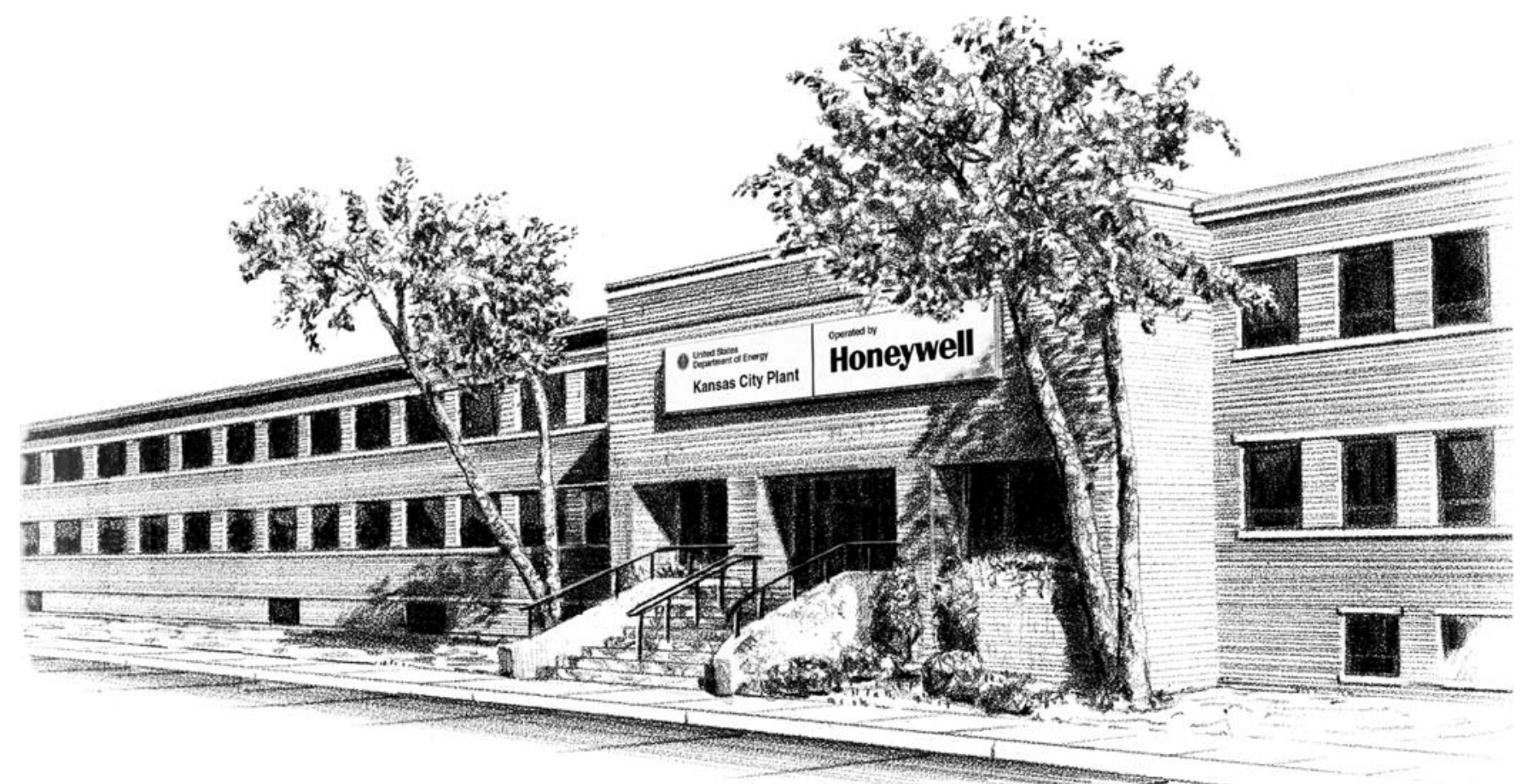

Prepared under prime contract DE-ACO4-01AL66850 for the

United States Department of Energy 


\section{DISCLAIMER}

This report was prepared as an account of work sponsored by an agency of the United States Government. Neither the United States Government nor any agency thereof, nor any of their employees, makes any warranty, express or implied, or assumes any legal liability or responsibility for the accuracy, completeness, or usefulness of any information, apparatus, product, or process disclosed, or represents that its use would not infringe privately owned rights. Reference herein to any specific commercial product, process or service by trade names, trademark, manufacturer, or otherwise, does not necessarily constitute or imply its endorsement, recommendation or favoring by the United States Government or any agency thereof. The views and opinions of authors expressed herein do not necessarily state or reflect those of the United States Government or any agency thereof.

All data presented, analyzed and presented has been developed in a specific context of work and was prepared for internal evaluation and use pursuant to that work authorized under the reference contract. Reference herein to any specific commercial product, process or service by trade name, trademark, manufacturer, or otherwise, does not necessarily constitute or imply its endorsement, recommendation or favoring by the United States Government, any agency thereof or Honeywell International, Inc.

Printed in the United States of America.

This report has been reproduced from the best available copy.

Available to DOE and DOE contractors from the Office of Scientific and Technical Information, P.O. Box 62, Oak Ridge, Tennessee 37831; prices available from (865) 576-8401, FTS 626-8401.

Available to the public from the National Technical Information Service, U.S. Department of Commerce, 5285 Port Royal, Rd., Springfield, Virginia 22161, (703) 487-4650.

A prime contractor with the United States Department of Energy under prime contract DE-ACO4-01AL66850.

\author{
Honeywell Federal Manufacturing \& Technologies \\ P. O. Box 419159 \\ Kansas City, Missouri 64141-6159
}




\section{Honeywell}

\section{KCP-613-8512 \\ Distribution Category UC-99}

Approved for public release; distribution is unlimited.

Selection and Implementation of a Replacement Cutting Tool

Selection Application

Gordon Rice

KCP-613-8512

September 2008

Final Report

Gordon Rice, Project Leader 


\section{Contents}

Section

Page

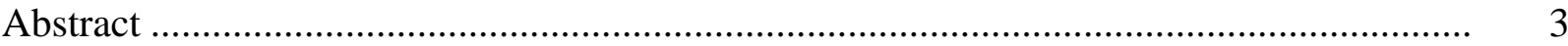

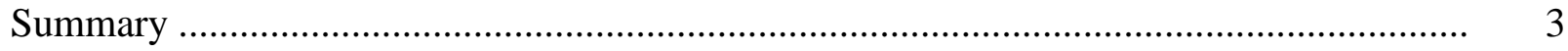

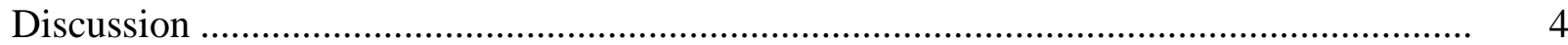

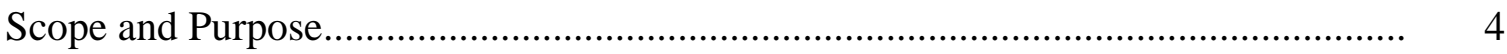

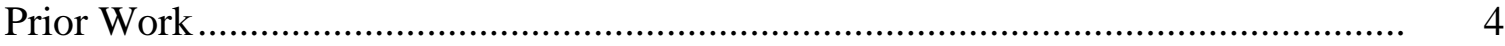

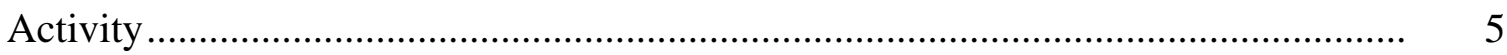

Phase One - Functional Requirements for the Replacement System ............... 5

Phase Two - Selection of a Replacement System...................................... 6

Phase Three - Existing Data Migration ................................................... 7

Phase Four - Data Validation, Configuration, and Training............................ 8

Phase Five - Individual Graphics Creation .............................................. 9

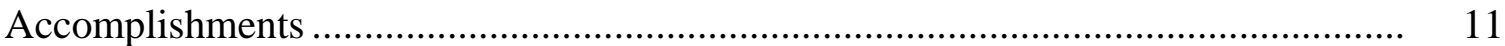

\section{Appendices}

A. Cutting Tool Database Interface Requirements ......................................... 13

B. SQL to Generate a Data File From the Existing System.................................

C. Sample Output From the SQL Export ........................................................ 58 


\section{Illustrations}

Figure Page

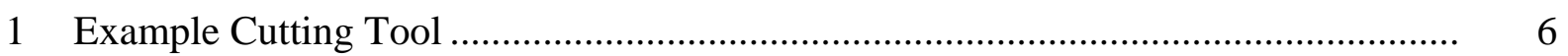

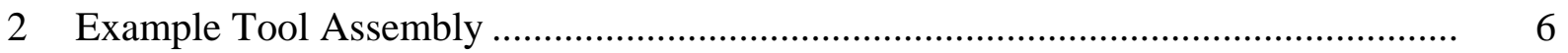

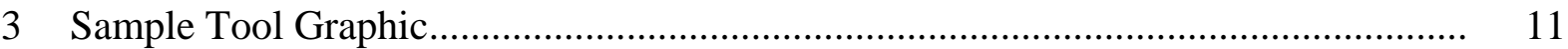

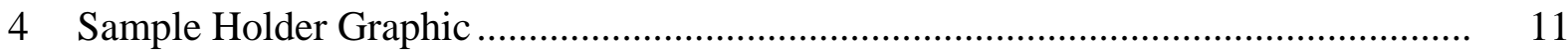




\begin{abstract}
A new commercial cutting tool software package replaced an internally created legacy system. This report describes the issues that surfaced during the migration and installation of the commercial package and the solutions employed. The primary issues discussed are restructuring the data between two drastically different database schemas and the creation of individual component graphics.
\end{abstract}

\title{
Summary
}

A custom built legacy system cutting tool application had become to expensive to maintain and needed replacement. A team evaluated replacement options of commercially available systems or a new custom system. After review, the commercial software option was implemented.

Structured Query Language (SQL) scripts were utilized during the migration and post migration validation steps. During data migration, code inside the SQL scripts translated data between fields and in some instances, created new data that did not exist within the old system. After the data was migrated, additional SQL scripts generated flat output to support data validation outside the new application interface.

The selected software was written and maintained by a German company. Language translation issues caused some relatively simple system configuration activities to become more time consuming. The delivered new system contained tool materials, part materials and unique mating codes to support automatic assembly of tools and holders. Virtually all of this supplied data in the original software required updating to make it compatible with KCP's terminology and standard materials.

A key element of the new application for user support was integration of cutting tool graphics within the application. Generation of the graphics to support this application was one of the more time consuming tasks in the entire process. Two approaches were employed: First, utilization of graphics wizard within the new software application. This tool was very useful for small families of tools, however, for large families, it was very time consuming. The second approach used ProEngineer and AutoCad. The flat validation text files were used to populate family tables in ProEngineer. After the family table was populated with data, a single ProEngineer solid model could quickly generate hundreds of unique graphics files. The output from ProEngineer was not directly usable by the application. A macro was created to run in AutoCad that made the final modifications required in the graphics files. 


\section{Discussion}

\section{Scope and Purpose}

An internally created cutting tool application had reached the end of its useful life and required replacement. This old legacy application was an internally created application that ran on top of some commercially created software development tools. The purpose of this project was to identify a replacement interface and support the development and deployment of the new interface. These activities were funded through 2008 ADAPT funding.

At the start of this project, possible solutions ranged from an internally created replacement interface to a complete replacement utilizing a commercially available package. The current state of the legacy system demonstrated the importance of locating a commercial package solution. Updating the system to current software development tools would require a start from scratch on the interface, a costly solution. During the creation of the legacy system commercial packages were very limited in capability. Searches that are more recent showed that far fewer packages were commercially available now.

The existing legacy cutting tool application supported process engineers primarily as a search tool for a wide range of cutting tool components. Due to the diverse range of products manufactured at the Kansas City Plant (KCP), the internal inventory system maintains over 4000 unique cutting tool definitions. Components range from single piece cutting tools to indexible components from many different commercial suppliers. Indexible cutting tools consist of a replaceable cutting edge, holder, and spare parts that are typically interchangeable only with a single manufacturer's product family. Locating a specific tool or finding mating indexible components is difficult without the aid of a custom search application.

\section{Prior Work}

A software search in 1998 had revealed six or seven commercial packages that supported cutting tool search and management software. The available packages at that time primarily focused on single point or single computer solutions. These commercial packages would not easily adapt to the distributed environment needed to support KCP's process engineering needs. The results of that evaluation led to the internal application development.

Internally created in 1998 and 1999, the legacy cutting tool application utilized a custom ORACLE database with custom interfaces that ran in ORACLE Forms and ORACLE Reports application software. The ORACLE database and structure were still very functional; however, the ORACLE Forms and ORACLE Reports applications were becoming unstable over time due to the changes to the underlying ORACLE software. An additional driver was that the pricing structure changes instituted by ORACLE 
(specifically Forms and Reports software) had dramatically increased the annual operating cost and was no longer a cost effective solution for the approximately 70 users.

\section{Activity}

The project was broken into several phases. Phase one involved a team of current application users to established functional requirements for a replacement system. Phase two used the same team to evaluate commercially available packages versus a possible internally created replacement system. Phase two's objective was to select the solution path. Phase three consisted of a small group of individuals defining the migration path for the existing legacy data and executing that migration activity. Phase four focused on validation of the migrated data, creation and delivery of a user training course for the new system users and configuring the software. The final phase, Phase five, focused on the creation of individual component graphics for all the unique cutting tool and holder components.

\section{Phase One - Functional Requirements for the Replacement System}

A small team of individuals that included the existing data owner, lead Cutting Tool Engineer, and other experienced users developed functional requirements for the replacement system. This team considered the old systems capabilities, level of usage of those capabilities, and noted deficiencies to develop new functional requirements. While the old system supported a wide range of capabilities a notable deficiency was its high difficulty of use. Capabilities not utilized in the old system were not included in the new system requirements. A copy of this original document is included in Appendix A.

The initial plan assumed that a commercial system capable of meeting the facilities needs did not exist. With this focus, the new requirements document used references to the existing database structure. The ORACLE database that contained the legacy application data is still a forward viable software tool. It would not require replacement if a new interface were developed. Incorporation of graphics was one key element that came out of the functional requirements development. If graphics could be included, the users wanted a highly capable system. If graphics would not be included, a relatively small set of functional requirements would survive.

If graphics were not included, Process Engineers required an application capable of the following:

- Locating a cutting tool to a user developed set of criteria (see Figure 1 for a sample cutting tool)

- Locating mating cutting tool components and offer those up to the user as a package meeting the query requirements.

If graphics were included, Process Engineers required an application capable of the following:

- Locating a cutting tool to user a developed set of criteria 
- Locating mating cutting tool components and offer those up to the user as a package meeting the query requirements

- Mating cutting tool components to machine holder components creating an assembly (see Figure 2 for a sample cutting tool assembly)

- Storing an assembly definition and assigning a unique ID number to the configuration

- Creating and storing a tool list (a group of assemblies that are used on a single part)

- Automatically generate a graphical picture of an assembly and tool list for inclusion in manufacturing work instructions.

- Ability to query for assemblies created by other Process Engineers

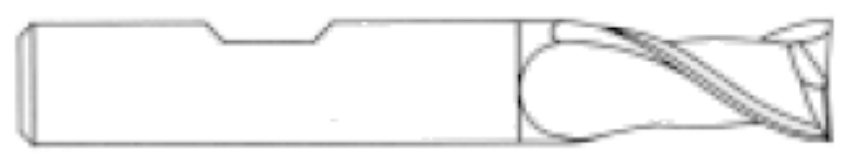

Figure 1 - Example Cutting Tool

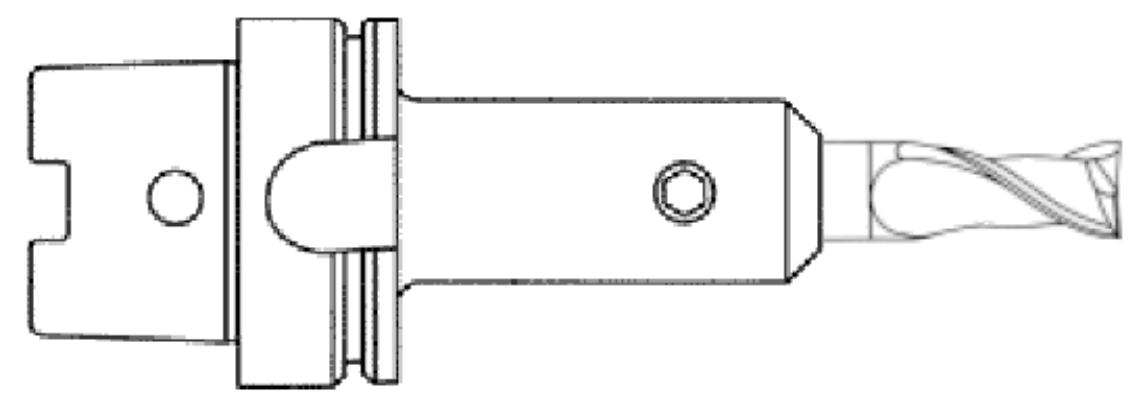

Figure 2 - Example Tool Assembly

A review of the requirements with Information Technology Team members identified limitations with an internally developed package. Experience with internally created legacy systems showed that custom systems needed to be very limited in scope. Large complex systems were difficult to maintain due to the frequent updates to commercial software and business needs. This lead the team to conclude that a commercial package would be required to deploy a highly capable system.

\section{Phase Two - Selection of a Replacement System}

The search for commercial cutting tool software tools resulted in identification of only two major systems that appeared to meet requirements and included graphics support. The 
two prospective systems are marketed in the US but are supported and maintained by German companies. Both systems appeared to be similar in capability and function from the user's viewpoint. A detailed review by Information Technology staff revealed that one of the systems was not current on adapting to software updates from Microsoft. This software package was subsequently removed from the potential list.

The second package went through additional reviews by the users and by Information Technology staff. The reviews became significantly more difficult because the company was relatively new to the United States and did not have any US citizens fully trained in the software operation and installation. After several telecoms and onsite visits, TDM Systems was selected and approved by both the users and the Information Technology group. The resulting cost to procure the software was determined to be roughly the same cost to develop an internally created but significantly less capable system.

\section{Phase Three - Existing Data Migration}

Migration of existing data into the new system began with a comparison of the legacy data structure to the new database structure. Evaluations of the new software during the prepurchase phase suggested that the table structures between the new and old systems were very compatible. The component information was displayed in very similar methods and contained much of the same information. After receipt of the software, it became clear that the storage strategies were quite different.

The design of the legacy system contained a unique table for each class of item. For example, cutting tools were stored in the cutting tool table, inserts in an insert table, spare parts in the spare part table and etc. Each row of the table contained the entire definition of the component. For flexibility, the tables contained fields that were variable and the contents of the field changed for different entities. Text Field 1 could contain thread pitch for a tap or a relief angle for an end mill. To cover all the components stored in the system seven unique tables contained component data and an additional five tables defined the variable fields in each table.

The new system contained all the component definitions in two tables. The primary table contained minimal information about the components like an identification number, name, quantity available, type of component and etc. This information was stored as a single row per component. The secondary table had only a small number of fields: the component identification number, a field identification value, and a single piece of information about the component. A third table contained a relationship between the component type and the field information value definition.

A migration strategy for the approximately 7000 individual components eventually evolved into a set of translation tables and a series of Structured Query Language (sql) text files. The central translation table contained a single row for each piece of information that was to be migrated. Essentially, each row defined the component type, the name of the source table, the name of the field, and the name of the destination field for a single piece of information. Using this approach allowed the query to make corrections or 
needed adjustments to the existing data without modifying the data in the existing system. The ability to leave the existing data unmodified was needed to support the ongoing use of the old system. See Appendix B for the query used to generate the detail data. The output generated by this query actually generated a new SQL file that would load the data into the new system. See Appendix C for a sample of this output file.

\section{Phase Four - Data Validation, Configuration, and Training}

Phase four activities focused on preparing the software for use by the users with the exception of the graphics creation. The first significant activity required manual validation of all the migrated data. System configuration followed when some usable data became available and completed with delivery of user training.

\section{Data Validation}

Data validation was originally planned as a relatively short-term activity since the data to be migrated was coming from a fully validated production system. After the migration was completed, it became clear that the validation task was significantly more complex. Two major topics drove this: First, the new system required cutting tools be broken down into much smaller functional groups than originally organized. Additionally, the new groups varied significantly from the old system. Secondly, support of the new more flexible query tools required additional database fields be populated. Some of this data did not exist in the legacy system.

Due to the large number of components that required validation, an approach external to the application was desired. A series of SQL queries were written that generated flat text output of the post-migrated data organized by tool groups. After importing these flat text files into Microsoft Excel, individuals validated the data. Changes were recorded directly back into the Excel file for later updates into the application. This approach allowed multiple individuals to perform validation prior to application deployment or application training.

Sample of the SQL scripts and Excel files are not included in Appendix. These files are unique to the database structure of the purchased application. Revealing the structure of the Excel files or the SQL scripts will reveal proprietary information from the software supplier.

\section{Configuration}

The software comes populated with a complete list of part materials, tool materials, tool groups, mating codes (how to match a cutting tool to a holder), and adapter definitions. During migration of existing data, it was clear that many of these supplier created data sets required configuration. Since the software supplier is a German based company they had populated these common tables with common European values. The European values are sufficiently different from US terminology as to make the values meaningless to most users. 
Tool and part material values were relatively straightforward to correct. The legacy system contained a full list of values from real user data. After deleting the supplied values, the legacy data was loaded into the system.

The system uses adapter definitions to mate tool assemblies (a unique combination of a cutting tool and one or more holders) to machines. Many of the European adapters are the same as those used in the United States, however, the European and US based names often vary. Configuring the adapter definitions included inserting new values from the legacy system as well as manually revising existing system names to match legacy system data.

Organizing the cutting tools and holders is supported in the new software through a series of Tool groups and subgroups. While many of the supplied tool group names aligned well with KCP accepted tool names, some were quite different. In some instances, system configuration required an entirely new subgroup to handle unique types of cutting tool used at KCP. A few Tool groups and subgroups simply required renaming to align with KCP accepted naming convention.

The new system utilizes mate codes to determine which cutting tools and holders will mate together. These codes are a key element of the systems ability to perform automatic tool assembly creation. Updating the mate codes required significantly more effort than any other configuration activity. Many of the standard US attachment approaches between cutting tools and holders are different from European systems. Additionally, for some types of connections like collets the system was setup to treat all collets as interchangeable. KCP utilizes collets procured over many years which has resulted in a wide variety of collet systems that do not mate. After developing a deep understanding of how the system performed mating, a method was implemented to support all of KCP's standard and unique attachment methods. Details are not included to prevent revealing software supplier proprietary information on how the system performs mating operations.

\section{User Training}

A representative from TDM Systems provided the initial onsite two-day user training to a small group of power users. One member of this initial training group developed the training outlines for the various internal user groups, which included groups of Data Owners, Engineers, Programmers, and Machinists. Training classes ranged from two hours to a full day for the various groups. The classroom trainer detailed out the actual classroom training and delivered user training to over 70 users of the new system.

\section{$\underline{\text { Phase Five - Individual Graphics Creation }}$}

The new cutting tool system supports individual graphics for each unique component. The unique graphics are not required, but do support several new capabilities over the legacy system. These include:

- User visual confirmation of the selected component, 
- Automatic generation of an assembly graphic,

- Size and shape data automatically fed into Vericut (a separate tool used in machining program validation)

The new software system supported an Autocad based DXF file format for graphics. Each graphic file contains views of the component on separate layers. The software is able to show the component by itself or in an assembly through internal manipulation of the visible layers. (See Figures 3 and 4 for examples of the graphics files)

Approach one focused on using ProEngineer computer aided modeling software to create the graphics. The concept involved creating a parameter driven graphic of the desired component family in ProEngineer. Data from the Microsoft Excel validation files drove the parameters and led to generation of unique graphics for each file. ProEngineer was not capable of placing all the required graphics components on the correct layers. This was resolved through the creation of a macro that runs inside Autocad. ProEngineer output files were modified inside Autocad with the macro leaving a usable graphic. This approach leveraged experienced ProEngineer users and the available Excel data. It was very effective for large component families where hundreds of tools could be created from one source ProEngineer file. For small component families, less than 50 tools, this was not a cost effective approach.

The second approach used a graphic creation tool purchased with the software. This tool created graphics ready for use inside the application; however, this tool did not support automated input. For each graphic created a user was required to manually input 10 to 20 unique numeric values correctly.

Both approaches were effective for the individual families of components they were applied to. The ProEngineer approach required a heavy investment of time up front to establish a parameter driven drawing for each component family; however, it was very efficient for individual component creation for large families. The ProEngineer approach also required an individual with extensive experience with ProEngineer. The internal software tool required only a few hours of experience for user training, but required manual input of all the unique component values. It was efficient for small component families. 


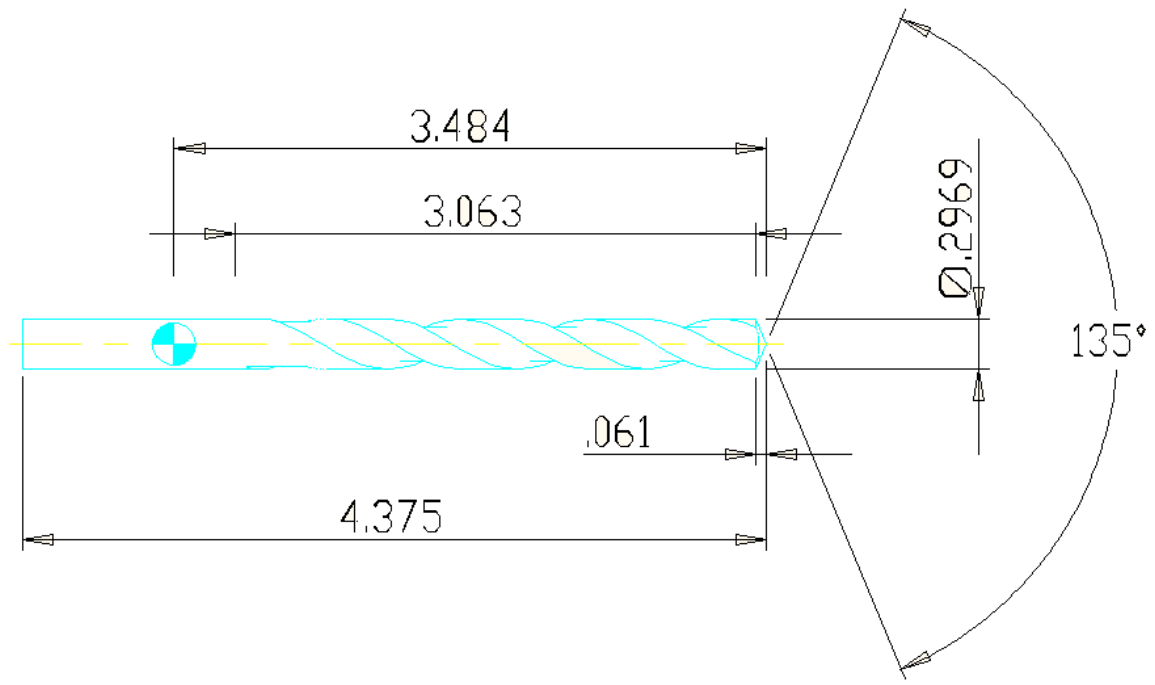

Figure 3 - Sample Tool Graphic

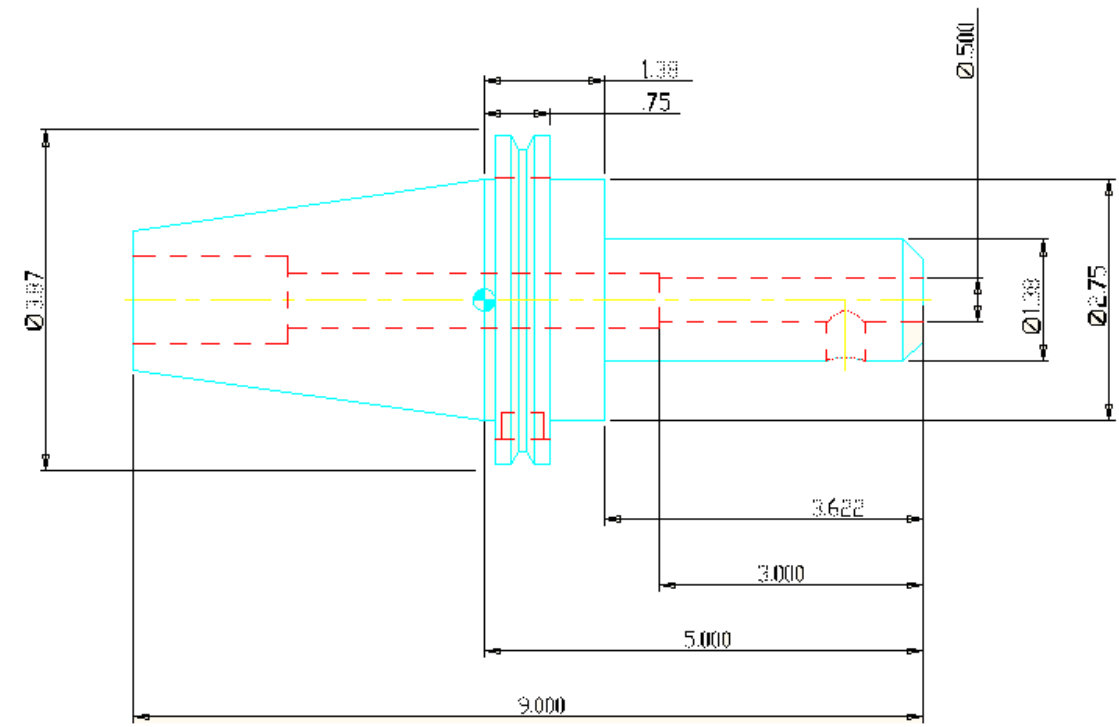

Figure 4 - Sample Holder Graphic

\section{Accomplishments}

A new cutting tool application was selected to replace an internally created legacy system. The following activities were completed prior to releasing the system for production use:

- Existing cutting tool information was migrated to the new system using custom generated SQL scripts.

- Additional custom SQL scripts were used to generate post migration data for validation efforts.

- Two methods of creating individual graphic files were evaluated and used to create graphics for approximately 3500 components. 
- User training for the new software was developed and deployed to over 60 plant users.

- Data provided by the software creator was reconfigured from European names and standards to support NWC tools and materials. 


\title{
Appendix A
}

\section{Cutting Tool Database Interface Requirements}

\author{
Gordon Rice, Paul A Biggs \& Richard Moreno \\ 10-15-2007
}

\section{General Description}

The cutting tool database is an application that maintains information on perishable and non perishable tooling aids in support of metal, plastic and other material machining operations. There are essentially two classes of information stored in the system, perishable and non perishable.

The perishable items are comprised of cutting tools, indexible inserts, indexible tooling spare parts and removable shanks for cutting tools. These items are considered perishable due to their close proximity to the machining operation. They either are in direct contact with the material being machined or are sufficiently close that they are at risk of damage or wear beyond use.

The non perishable items are comprised of milling holders, extensions, collets and other machine specific adapters. The purpose of these devices is to act as the interface between the cutting tool and the machine that is being used. They are considered non perishable because the risk of damage or wear is significantly lower due to being farther from the machining. Honeywell FM\&T currently maintains an application that uses an Oracle Database with an Oracle Forms and Oracle Reports Interface. The current Database Table structure is shown in the section titled "Assembly Database Table Structure". Some information about the contents of the current database is included in the section titled "Sample Database Table Contents".

Two potential paths are clear for a new Cutting Tool Interface. One option is to purchase an off the shelf commercial package that performs the required functions. The second path is to define and create a new interface based on our existing information and database structure. A commercial package has the potential to provide significantly more capability as the implementation costs are shared among all users of the commercial package. In an effort to define requirements for both approaches, this document is being broken into three major requirements sections. The first group is called Rollout Mandatory Requirements, followed by Mandatory Requirements followed by Desirable Requirements.

Rollout Mandatory Requirements are those functions that are required to be implemented before the current Forms and Reports application can be retired. These represent functions that are either used on a daily or weekly basis, or functions that are required to maintain database information related to those high use items. Mandatory Requirements are features that are implemented in the current interface, but are used at a lower rate and could be implemented over a longer period of time. These were heavily used by a department within FM\&T, but are currently only lightly used by other manufacturing departments. Their lower usage rate is primarily due to the current interface being difficult to use in these areas. Mandatory Requirements can be rolled out up to six months after the current interface is shut down.

Desirable Requirements represent capabilities that were not incorporated into the existing interface, but would be highly useful to the application. A few of these are identified as deficiencies in the scope of the current application but are relatively easy to incorporate. Most of these requirements represent capabilities incorporated into commercially available packages that could replace the existing application.

\section{General Requirements}

\section{Units}

The system shall be capable of supporting both English and Metric Units or alternately, it must be capable of supporting all units in English. If components can be specified in either English or Metric units, the system must be capable of mating components with different units with the results reflecting the correct size of the mated components. For example, a metric drill will fit 
into an English collet as long as the metric drill size fits within the defined range of sizes for the collet.

\title{
Interface
}

The ability to have multiple interface screens active. For example, when entering a new cutting tool, it may be determined that a new Tool Type, Tool Style is required. The interface will allow the user to retain their current efforts, go the required sub screen, make the required updates or inserts to support the new entry, then return to the original entry screen and complete the entry. It is desirable that any graphics and reports be directly reachable from within the interface. For example, if a user queries for information within the interface, the contents of the query can be directly passed to the report for output in multiple formats.

\section{Database Fields}

To proceed forward with a procured off the shelf package, additional information should be added in each section identifying the minimum fields that are required for each item to retain. This is less significant for an application that is created on top of the existing KCP database, as the required fields are already defined in the database.

\section{Multiple User Roles}

The new interface must support multiple user role with varying permission levels for each individual role.

- IT administrator - Full permissions

- Application Administrator- Full read and edit capability on all table contents. Essentially the equivalent of a Cutting Tool Engineer and a Selected Process Engineer rolled into one role. Probably a Lead Process Engineer.

- Cutting Tool Engineer -Edit capability on Cutting Tools, Inserts, Removable Shanks, and the related fields and tables to support these items. This would include the items in the Cutting Tool Maintenance section. See the detail sections on these items for related tables.

- Selected Process Engineer - All capabilities as the role Process Engineer, with the ability to Insert, Update and Delete in the Holders, Extensions and Collets sections. See the detail section on these items for related tables.

- Process Engineer - Insert, Update and Delete capabilities on Tool Assemblies, Tool Lists and the related support tables. See the detail sections on these items for related tables. Full Query capability, Create tool assemblies, tool lists and reports

- NC Analyst -Same access as a Process Engineer

- Manufacturing Crib Attendant - Insert, Update and Delete capabilities on the departments and quantities of available components for Holders, Extensions and Collets. Full read and query capability, with report generation

- All Users - Read access to the entire interface and table structure with full query capability.

\section{Rollout Mandatory Requirements}

Requirements listed in this section represent the minimum capabilities that must exist in a new or replacement interface before the current Oracle Forms and Oracle Reports interface may be deactivated and retired.

\section{Cutting Tools}

\author{
Inserting, Updating and Deleting \\ Role - Cutting Tool Engineer
}


Interactive screens that allow a cutting tool engineer the ability to insert, update and delete Cutting Tools. This screen will also contain a link or path to the definition for connections to matching Inserts.

Each Cutting Tool is uniquely defined by a Cutting Tool Number. The number is assigned by the individual creating the tool and is at least an 8 digit field and up to a 14 character alpha numeric field that can contain a hyphen.

Tool Types and Tool Styles will be validated through a list of available styles.

For most character based fields, the inserted values shall be validated to an authorized list of values. Examples would be Hand of Cut, Tool Material, Spiral Direction, Cutting Edge Style, and Shank Style.

The types of information required to define a cutting tool vary from Tool Style to Tool Style. The application shall support a method of handling these varying attributes such that it is seamless to the user as well as supporting returning multiple Tool Styles from a single query.

Access to Insert, Update and Delete Cutting Tool definitions shall be limited to a specific group of users. These are Cutting Tool Engineers and an application Administrator.

The application will maintain a list of who and when a user modified a definition of a cutting tool. Support the user setting up the information required to determine which inserts will fit into indexible cutting tools. The system shall be capable of supporting at least three different insert types for each tool. Identify the quantity of pockets for each insert type.

Support connections required to mate cutting tools that require removable shanks to the mating removable shank.

Support connections to mate a cutting tool to a Holder, Extension or Machine adapter.

Support a user generated list of Spare Parts identified by the user generated Spare Part Id number and the quantity required for each item. For example, shims, screws, wrenches, clamps, etc.

Support the inserting and maintenance of experimental items. These tools are intended to only exist in the database for a time of one year or less. Allow the experimental tools to be identified as experimental and thus not available for general use. Support automatic notification to Cutting Tool Engineering of experimental tooling still in the database that is over 6 months old and subsequent notices on one month intervals.

The system must be user configurable to allow defining new cutting tool types and styles. When defining new tool types and styles, the user must also have the ability to setup the new unique attribute fields for the new tool type and style along with setting up any list of value fields for the new attributes and forced validation on those list of value fields.

Prevent deletion if the item is used in a Tool Assembly.

\section{Additional Information if the current Database Tables are used.}

The primary database table in the current application is CUTTING_TOOLS. The Cutting Tool Table contains 14 fields that the name and contents change for each cutting tool based on the Tool Type and Tool Style.

Secondary Tables are:

CTOOL_INSERT_XREFS - These table contents are set for the Cutting Tool only if the Cutting Tool uses indexible inserts. In these instances, the contents of the table define which Indexible Inserts will fit into the Cutting Tool, the quantity required and the location of the pocket.

INSERT_LINK_CODES - This table lists the available codes to match a Cutting Tool to an Insert.

TOOL_STYLE - This table lists the authorized list of Tool Type and Tool Style combinations.

TOOL_ATTS - This table lists the name of each of the variable fieldnames for the 14 variable fields based on Tool Type and Tool Style.

SPARE_PART_XREFS - For cutting tools that use spare or replacement parts, this table lists the parts for each cutting tool.

LOV_TABLES - This table lists all of the available values for each list of value. 
LOV_NAMES - This table lists all of the List of Value fields that are enforced within the application along with a definition of the field.

FIELD_MODIFICATIONS - Records who, when and why a cutting tool definition was revised.

TOOL_NUMBERS - This table is used to force unique ID numbers in all the tables, INSERTS, SPARE_PARTS, CUTTING_TOOLS, \& REMOVABLE_SHANK_ARBORS

\section{Query For Cutting Tools}

\section{Role - All Users}

Query for Cutting Tools with the ability to limit return on at least the following information.

Cutting Tool Number, specific or with wildcards.

Tool Type

Tool Style

Cut diameter, specific size or range.

Cutting Tool Material

Cutting Tool Coating

Cutting Edge Style

Length of Cut, specific size or range.

Overall Length, specific size or range.

For character fields, support exact matches and wildcard matches

For numeric fields, support exact matches, minimum limits, maximum limits, and range values. For character fields with a list of values, offer the list of available values to aid the user.

\section{Reporting}

Role - All Users

Support reports that output the results from the query. The report shall include all the components that make up an entry meeting the query criteria, along with the major fields for each component. User customizable is preferred.

Support output in the following formats:

Printed Paper

PDF

TIFF

JPG

CSV (Import into Excel)

\section{Inserts}

\section{Inserting, Updating and Deleting}

Role - Cutting Tool Engineer

Interactive screens that allow a cutting tool engineer the ability to insert, update and delete Inserts. This screen will also contain a link or path to the definition for connections to matching Cutting Tools.

Each Insert is uniquely defined by an Insert Tool Number. The number is assigned by the individual creating the tool and is at least an 8 digit field and up to a 14 character alpha numeric field that can contain a hyphen.

For most character based fields, the inserted values shall be validated to an authorized list of values.

Access to Insert, Update and Delete Insert definitions shall be limited to a specific group of users. These are Cutting Tool Engineers and an application Administrator. 
The application will maintain a list of who and when a user modified a definition of a insert.

Support the user setting up the information required to control which inserts will fit into indexible cutting tools.

Support the inserting and maintenance of experimental items. These tools are intended to only exist in the database for a time of one year or less. Allow the experimental tools to be identified as experimental and thus not available for general use. Support automatic notification to Cutting Tool Engineering of experimental tooling still in the database that is over 6 months old and subsequent notices on one month intervals.

The system must be user configurable to allow defining new insert types. When defining new insert types, the user must also have the ability to add any values to list of value fields to support forced validation.

Prevent deletion if the item is used in a Tool Assembly.

\section{Additional Information if the current Database Tables are used.}

The primary database table in the current application is INSERTS.

Secondary Tables are:

INSERT_LINK_CODES - This table lists the available codes to match a Cutting Tool to an Insert.

LOV_TABLES - This table lists all of the available values for each list of value.

LOV_NAMES - This table lists all of the List of Value fields that are enforced within the application along with a definition of the field.

FIELD_MODIFICATIONS - Records who, when and why a cutting tool definition was revised. TOOL_NUMBERS - This table is used to force unique ID numbers in all the tables, INSERTS, SPARE_PARTS, CUTTING_TOOLS, \& REMOVABLE_SHANK_ARBORS

\section{Query}

Role - All Users

Query for Inserts with the ability to limit return on at least the following information.

Insert Number, specific or with wildcards.

Insert Style

Insert Link Code

Insert Material

Insert Coating

Edge Radius

Insert Type

For character fields, support exact matches and wildcard matches

For numeric fields, support exact matches, minimum limits, maximum limits, and range values.

For character fields with a list of values, offer the list of available values to aid the user.

\section{Reporting}

Role - All Users

Support reports that output the results from the query. The report shall include all the components that make up an entry meeting the query criteria, along with the major fields for each component. User customizable is preferred.

Support output in the following formats:

Printed Paper

PDF

TIFF

JPG 
CSV (Import into Excel)

\title{
Removable Shanks
}

\section{Inserting, Updating and Deleting}

\author{
Role - Cutting Tool Engineer
}

Interactive screens that allow a cutting tool engineer the ability to insert, update and delete Removable Shanks. This screen will also contain a link or path to the definition for connections to matching Cutting Tools.

Each Removable Shank is uniquely defined by a Removable Shank Tool Number. The number is assigned by the individual creating the tool and is at least an 8 digit field and up to a 14 character alpha numeric field that can contain a hyphen.

For most character based fields, the inserted values shall be validated to an authorized list of values.

Access to Insert, Update and Delete Removable Shank definitions shall be limited to a specific group of users. These are Cutting Tool Engineers and an application Administrator.

The application will maintain a list of who and when a user modified a definition of a Removable Shank.

Support the user setting up the information required to control which Removable Shanks will fit mate with cutting tools.

Support a user generated list of Spare Parts identified by the user generated Spare Part Id number and the quantity required for each item.

Support the inserting and maintenance of experimental items. These tools are intended to only exist in the database for a time of one year or less. Allow the experimental tools to be identified as experimental and thus not available for general use. Support automatic notification to Cutting Tool Engineering of experimental tooling still in the database that is over 6 months old and subsequent notices on one month intervals.

The system must be user configurable to allow defining new removable shank types. When defining new removable shank types, the user must also have the ability to add any values to list of value fields to support forced validation.

Prevent deletion if the item is used in a Tool Assembly.

Additional Information if the current Database Tables are used.

The primary database table in the current application is REMOVABLE_SHANK_ARBORS. Secondary Tables are:

SHANK_LINK_CODES - This table lists the available codes to match a Cutting Tool to a Removable Shank.

LOV_TABLES - This table lists all of the available values for each list of value.

LOV_NAMES - This table lists all of the List of Value fields that are enforced within the application along with a definition of the field.

FIELD_MODIFICATIONS - Records who, when and why a cutting tool definition was revised.

TOOL_NUMBERS - This table is used to force unique ID numbers in all the tables, INSERTS, SPARE_PARTS, CUTTING_TOOLS, \& REMOVABLE_SHANK_ARBORS

\section{Query}

Role - All Users

Query for Removable Shanks with the ability to limit return on at least the following information.

Removable Code Number, specific or with wildcards.

Shank Style 
Shank Link Code or Shank Xref

Shank Material

Shank Type

For character fields, support exact matches and wildcard matches

For numeric fields, support exact matches, minimum limits, maximum limits, and range values.

For character fields with a list of values, offer the list of available values to aid the user.

\section{Reporting}

Role - All Users

Support reports that output the results from the query. The report shall include all the components that make up an entry meeting the query criteria, along with the major fields for each component. User customizable is preferred.

Support output in the following formats:

Printed Paper

PDF

TIFF

JPG

CSV (Import into Excel)

\section{Cutting Tool Maintenance}

\section{Maintain Tool Styles}

Inserting, Updating and Deleting

Role - Cutting Tool Engineer

Allow Cutting Tool Engineers access to maintain the list of Tool Types and Tool Styles. For each Tool Style, support maintaining a list of variable field names unique to the Tool Type and Tool Style. When changing or adding a Tool Style, defining or adjusting the variable field attributes will also be supported.

Additional Information if the current Database Tables are used.

The primary database tables in the current application is TOOL_STYLE and TOOL_ATTS.

Restrict Deletion if the Tool Style is used in an existing cutting tool definition.

Secondary Tables are:

LOV_TABLES - This table lists all of the available values for each list of value.

LOV_NAMES - This table lists all of the List of Value fields that are enforced within the application along with a definition of the field.

\section{Maintain List of Values}

\section{Inserting, Updating \& Deleting}

Role - Cutting Tool Engineer and Selected Process Engineers (List of Values Table also support Holders, Extensions and Collets)

Allow Cutting Tool Engineers access to maintain the list of table or tables that maintain lists of values that new or updated fields are validated against.

Additional Information if the current Database Tables are used.

The primary database tables in the current application is LOV_NAMES, and LOV_TABLES. 


\section{Maintain Insert Link Codes}

\section{Inserting, Updating and Deleting}

Role - Cutting Tool Engineer

Allow Cutting Tool Engineers access to maintain the values used for Insert to Cutting Tool linkages. They shall be based on ANSI Insert definitions and be user customizable. Fields shall include sufficient information for the user to determine what inserts mate to the code.

Additional Information if the current Database Tables are used.

The primary database table in the current application is INSERT_LINK_CODES.

\section{Maintain Shank Link Codes}

Inserting, Updating and Deleting

Role - Cutting Tool Engineer

Allow Cutting Tool Engineers and access to maintain the values used for Cutting Tool to Removable Shank linkages. The application shall maintain the list of codes, along with fields that include the Type, Manufacturer, Size information, ANSI Code, and Hand of the shank. These codes shall be user customizable.

Additional Information if the current Database Tables are used.

The primary database table in the current application is SHANK_LINK_CODES.

\section{Maintain Spare Parts}

\section{Inserting, Updating and Deleting}

Role - Cutting Tool Engineer

Allow Cutting Tool Engineers and access to maintain the Spare Parts List. Spare Parts shall be defined with at least a user defined unique ID number up to 14 characters alphanumeric, text description of 20 characters, manufacturer, and manufacturer catalog number.

Additional Information if the current Database Tables are used.

The primary database table in the current application is SPARE_PARTS.

Secondary Tables are:

LOV_TABLES - This table lists all of the available values for each list of value.

LOV_NAMES - This table lists all of the List of Value fields that are enforced within the application along with a definition of the field.

FIELD_MODIFICATIONS - Records who, when and why a cutting tool definition was revised.

\section{Cutting Tool Selection}

\section{Query}

Role - All Users

Provide a query screen that allows the user to input a wide range of requirements for a cutting tool and return a list of tools meeting the criteria back to the user. The results of the query shall offer all combinations of solid cutting tools, indexible cutting tools with mating inserts and cutting tools requiring removable shanks along with the mating shank based on the query criteria.

Query criteria shall include Tool Type, Tool Style, Length of Cut, Hand of Cut, Tool Material (If indexible, based on the insert), Tool Coating (If indexible, based on the insert), Shank Type and Shank Sizes. For rotating tooling, also allow query on Tool Diameter. For turning tooling, also allow query on min cut diameters, tip orientation, and etc. For character fields, support drop downs and wild cards. For numeric fields, support exact values and range values. 
Allow the user to modify or adjust an entered query to fine tune the results without requiring the user to start from scratch. For example, if a query is run and no tools are returned the user may wish to modify a single value or remove one entry, or if many tools are returned the user may want to adjust to be more selective.

\section{Reporting}

Role - All Users

Support reports that output the results from the query. The report shall include all the components that make up an entry meeting the query criteria, along with the major fields for each component. User customizable is preferred.

\section{Additional Information if the current Database Tables are used.}

The primary database tables in the current application are CUTTING_TOOLS, INSERTS, CTOOL_INSERT_XREFS, REMOVABLE_SHANK_ARBORS, TOOL_ATTS, TOOL_STYLE.

\section{Mandatory Requirements}

Requirements listed in this section represent lesser used functionality in the current interface that must be implemented, but can be rolled out after the current interface is turned off.

\section{Holders}

\section{Inserting, Updating and Deleting}

Role - Selected Process Engineer

Interactive screens that allow selected process engineers the ability to insert, update and delete Holders.

Each Holder is uniquely defined by a Holder Id number. The number is assigned by the individual creating the item and is capable of supporting at least a 4 digit alphanumeric field. Longer up to 14 characters is preferred.

For most character based fields, the inserted values shall be validated to an authorized list of values.

Access to Insert, Update and Delete Holder definitions shall be limited to a specific group of users. These are a select group of Process Engineers and an application Administrator.

The application will maintain a list of who and when a user modified a definition of a Holder.

Support the user setting up the information required to control the information to determine if a Holder will mate with items like collets, extensions and cutting tools.

The system must be user configurable to allow defining new holder types. When defining new holder types, the user must also have the ability to:

- Add field definitions for variable field names matching the new holder type

- Add any values to list of value fields to support forced validation.

Prevent deletion if the item is used in a Tool Assembly.

\section{Maintain Departmental Quantities}

Role - Selected Process Engineers \& Manufacturing Crib Attendant

Maintain the quantity of a specific item in a department and the department number the items are located.

\section{Additional Information if the current Database Tables are used.}

The primary database table in the current application is HOLDERS

Secondary Tables are: 
HOLDER_DEPTS - Contains the departments that currently maintain copies of this item and the quantity owned by the department.

HOLD_EXT_TYPES - This table defines the various types of Holders that are available in the system.

HOLD_EXT_ATTS - This table defines the names of the variable fields in the Holders table based on the Hold_Ext_Type that is defined for the Holder.

PULL_ID - Table that contains a list of new unique IDs for new holder and extensions. New defined items are selected from the available values.

LOV_TABLES - This table lists all of the available values for each list of value.

LOV_NAMES - This table lists all of the List of Value fields that are enforced within the application along with a definition of the field.

\section{Query}

Role - All Users

Query for Holders with the ability to limit return on at least the following information.

Holder Id

Mating Feature to the Machine

Mating Feature on the Cutting Tool side (size and method).

Type of Holder

At least Length and Width/Diameter

Manufacturing department owning the item

Quantity available in each department

For character fields, support exact matches and wildcard matches

For numeric fields, support exact matches, minimum limits, maximum limits, and range values.

For character fields with a list of values, offer the list of available values to aid the user.

\section{Reporting}

Role - All Users

Support reports that output the results from the query. The report shall include all the components that make up an entry meeting the query criteria, along with the major fields for each component. User customizable is preferred.

Support output in the following formats:

Printed Paper

PDF

TIFF

JPG

CSV (Import into Excel)

\section{Extensions}

\section{Inserting, Updating and Deleting}

Role - Selected Process Engineer

Interactive screens that allow selected process engineers the ability to insert, update and delete Extensions.

Each Extension is uniquely defined by a Extension Id number. The number is assigned by the individual creating the item and is capable of supporting at least a 4 digit alphanumeric field. Longer up to 14 characters is preferred. 
For most character based fields, the inserted values shall be validated to an authorized list of values.

Access to Insert, Update and Delete Extension definitions shall be limited to a specific group of users. These are a select group of Process Engineers and an application Administrator.

The application will maintain a list of who and when a user modified a definition of a Extension.

Support the user setting up the information required to control the information to determine if a Extension will mate with items like collets and cutting tools.

The system must be user configurable to allow defining new extension types. When defining new extension types, the user must also have the ability to:

- $\quad$ Add field definitions for variable field names matching the new extension type

- $\quad$ Add any values to list of value fields to support forced validation.

Prevent deletion if the item is used in a Tool Assembly.

\section{Maintain Departmental Quantities}

Role - Selected Process Engineers \& Manufacturing Crib Attendant

Maintain the quantity of a specific item in a department and the department number the items are in.

\section{Additional Information if the current Database Tables are used.}

The primary database table in the current application is EXTENSIONS

Secondary Tables are:

EXTENSION_DEPTS - Contains the departments that currently maintain copies of this item and the quantity owned by the department.

HOLD_EXT_TYPES - This table defines the various types of Extensions that are available in the system.

HOLD_EXT_ATTS - This table defines the names of the variable fields in the Extension table based on the Hold_Ext_Type that is defined for the Extension.

EXT_TAIL_TYPES - This table defines the various types of Extension ends that are available in the system.

EXT_TAIL_ATTS - This table defines the names of the variable fields in the Extension table based on the Ext_Tail_Type that is defined for the Extension.

PULL_ID - Table that contains a list of new unique IDs for new holder and extensions. New defined items are selected from the available values.

LOV_TABLES - This table lists all of the available values for each list of value.

LOV_NAMES - This table lists all of the List of Value fields that are enforced within the application along with a definition of the field.

\section{Query}

Role - All Users

Query for Extensions with the ability to limit return on at least the following information.

Extension Id

Mating Feature on the non cutting tool side (size and method).

Mating Feature on the Cutting Tool side (size and method).

Type of Extension

At least Length and Width/Diameter

Manufacturing department owning the item

Quantity available in each department

For character fields, support exact matches and wildcard matches 
For numeric fields, support exact matches, minimum limits, maximum limits, and range values. For character fields with a list of values, offer the list of available values to aid the user.

\section{Reporting}

Role - All Users

Support reports that output the results from the query. The report shall include all the components that make up an entry meeting the query criteria, along with the major fields for each component. User customizable is preferred.

Support output in the following formats:

Printed Paper

PDF

TIFF

JPG

CSV (Import into Excel)

\section{Collets}

\section{Inserting, Updating and Deleting}

Role - Selected Process Engineer

Interactive screens that allow selected process engineers the ability to insert, update and delete Collets.

Each Collet is uniquely defined by a Collet Id number. The number is assigned by the system or alternately by the user.

For most character based fields, the inserted values shall be validated to an authorized list of values.

Access to Insert, Update and Delete Extension definitions shall be limited to a specific group of users. These are a select group of Process Engineers and an application Administrator.

The application will maintain a list of who and when a user modified a definition of a Collet.

Support the user setting up the information required to control the information to determine if a Collet will mate with items like holders, extensions and cutting tools.

The system must be user configurable to allow defining new collet types. When defining new collet type, the user must also have the ability to add any values to list of value fields to support forced validation.

Prevent deletion if the item is used in a Tool Assembly.

\section{Maintain Departmental Quantities}

Role - Selected Process Engineers \& Manufacturing Crib Attendant

Maintain the quantity of a specific item in a department and the department number the items are in.

\section{Additional Information if the current Database Tables are used.}

The primary database table in the current application is COLLETS

Secondary Tables are:

COLLET_DEPTS - Contains the departments that currently maintain copies of this item and the quantity owned by the department.

LOV_TABLES - This table lists all of the available values for each list of value.

LOV_NAMES - This table lists all of the List of Value fields that are enforced within the application along with a definition of the field. 


\section{Query}

Role - All Users

Query for Collets with the ability to limit return on at least the following information.

Collet Id

Mating Feature on the non cutting tool side (size and method).

Mating Feature on the cutting tool side (size and method).

Type of Collet

Manufacturing department owning the item

Quantity by department

For character fields, support exact matches and wildcard matches

For numeric fields, support exact matches, minimum limits, maximum limits, and range values.

For character fields with a list of values, offer the list of available values to aid the user.

\section{Reporting}

Role - All Users

Support reports that output the results from the query. The report shall include all the components that make up an entry meeting the query criteria, along with the major fields for each component. User customizable is preferred.

Support output in the following formats:

Printed Paper

PDF

TIFF

JPG

CSV (Import into Excel)

\section{Tool Assemblies}

\section{Inserting, Updating and Deleting}

\section{Role - Process Engineers and NC Analyst}

Interactive screens that allow process engineers the ability to insert, update and delete Tool Assemblies.

Each Tool Assembly is uniquely defined by an Id number. The number is assigned by the system or alternately by the user. Preferred method is to assign a unique number from a range of values where the range is reserved for a assembly type. For example, all drilling assemblies would be grouped together.

For most character based fields, the inserted values shall be validated to an authorized list of values.

The application will maintain a list of who and when a user modified a definition of an Assembly. The system must be user configurable to allow defining new Tool Assembly Application types. However, creation of a new application type will be restricted to select process engineers.

A completed tool assembly shall contain all the components required to attach a cutting tool to the machine. This would include the Holder, any extensions, any collets required for the holder or the extension, the cutting tool, any inserts, any removable shanks.

Support the user selecting a Tool Assembly, copying that Assembly and then modifying the new copy to save under a new Id number.

Prevent deletion if the item is used in a Tool List 


\section{Create Tool Assemblies}

Role - Process Engineers and NC Analyst

The interface shall aid the user during the process of creating a Tool Assembly.

1. Select a cutting tool. Utilize a process similar or identical to the process defined in the section "Cutting Tool Selection". Essentially allow the user to input information about the cutting tool that they need; the system will offer back tools meeting the criteria. The offered list shall include solid cutting tools as well as indexible cutting tools, mating inserts, and, if required, mating removable shanks to the user. The select combination will be saved against the Tool Assembly. Some cutting tools use more than one insert; support selecting the additional inserts if needed.

2. Some assemblies can have multiple cutting tools. Support selecting additional cutting tools.

3. Using the selected cutting tool, allow the user to select any extensions and if required the mating collets. The interface should aid the user in locating mating components as well as supporting querying for mating extensions that meet criteria like the Query requirements defined under the section titled Extensions.

4. Some tool assemblies utilize multiple extensions where the prior extension mates into the extension. The interface should aid the user in locating mating components as well as supporting querying for mating extensions that meet criteria like the Query requirements defined under the section titled Extensions.

5. Tool Assemblies end in a Holder that mates to the machine. The interface should aid the user in locating mating components as well as supporting querying for mating holders that meet criteria like the Query requirements defined under the section titled Holders.

6. The interface shall aid the user in completing the additional fields that define a tool assembly. These include the overall length of the entire assembly, cutting diameter if a rotating tool and other fields.

\section{Additional Information if the current Database Tables are used.}

The primary database tables in the current application are TOOL_ASSEMBLIES, ASSEMBLY_TOOLS, INSERT_USAGES, EXTENSION_USAGES, TOOL_ASSY_NOS

Secondary Tables are:

All tables associated with Cutting Tools

All tables associated with Inserts

All tables associated with Spare Parts

All tables associated with Removable Shanks

All tables associated with Holders

All tables associated with Extensions

All tables associated with Collets

LOV_TABLES - This table lists all of the available values for each list of value.

LOV_NAMES - This table lists all of the List of Value fields that are enforced within the application along with a definition of the field.

Current database may function better on creating a tool assembly if some additional fields are added to the tables. For example, additional link codes could be created to identify mating conditions between holders and extensions, holders and tools etc. Additional fields like this could reduce the effort to support mating connections in the interface, thus reducing interface complexity and coding time.

\section{Query}

Role - All Users 
Query for Tool Assemblies with the ability to limit return on at least the following information.

Assembly Id

Interface to machine

Tool Assembly Type

Tool Assembly Gage Length

Holder Type

Cutting Tool Type and Style

Cutting Tool ID

Cutting Tool material (for indexible tools, this is the Insert material)

Cutting Tool Diameter

Cutting Tool Edge Type

Department the item can be built in

For character fields, support exact matches and wildcard matches

For numeric fields, support exact matches, minimum limits, maximum limits, and range values.

For character fields with a list of values, offer the list of available values to aid the user.

\section{Reporting}

Role - All Users

Support reports that output the results from the query. The report shall include all the components that make up an entry meeting the query criteria, along with the major fields for each component. User customizable is preferred.

Support output in the following formats:

Printed Paper

PDF

TIFF

JPG

CSV (Import into Excel)

\section{Tool Lists}

\section{Inserting, Updating and Deleting}

Role - Process Engineers and NC Analyst

Interactive screens that allow process engineers the ability to insert, update and delete Tool Lists.

Each Tool List is uniquely defined by an Id number. The number is assigned by the system or alternately by the user. Preferred method is to assign a unique number.

For most character based fields, the inserted values shall be validated to an authorized list of values.

The application will maintain a list of who and when a user modified a Tool List.

Tool Lists shall have a status field that identifies them as released or not released status. When released, the contents of the Tool List may not be altered. Provide a mechanism to create a copy of a Tool List or to move a Tool List from released to an editable state.

\section{Additional Information if the current Database Tables are used.}

The primary database tables the current application are PART_OPERATIONS, ASSEMBLY_USAGES, ASSEMBLY_USES, MATERIALS, MATERIAL_GROUPS, MAIN_MATERIALS

Feed and Speed tables are: 
CUT_PARM_USAGES, REC_FEED_SPEEDS, MATERIALS, MATERIAL_GROUPS, MAIN_MATERIALS

Secondary Tables are:

All tables associated with Tool Assemblies

All tables associated with Cutting Tools

All tables associated with Inserts

All tables associated with Spare Parts

All tables associated with Removable Shanks

All tables associated with Holders

All tables associated with Extensions

All tables associated with Collets

LOV_TABLES - This table lists all of the available values for each list of value.

LOV_NAMES - This table lists all of the List of Value fields that are enforced within the application along with a definition of the field.

\section{Feeds and Speeds}

\section{Role - Process Engineers and NC Analyst}

The systems shall provide screens for process engineers to enter and maintain feeds and speeds for Tool Assemblies. The feeds and speeds shall also be related to a specific part or to a specific machined material. The ability to enter and maintain multiple feeds and speeds for the same tool and material is required along with the ability to name the specific feed and speed.

Stored Feeds and Speeds may originate from a general area (for example, MetCut) or from existing usage (similar assembly from an existing tool list). Feed and Speed information shall include feedrate in Inches per minute or Inches per tooth, cutting speed in revolutions per minute or surface feet per minute, and depth of cut (both radial and axial values).

The interface shall have screens allowing users to locate other relevant feeds and speeds when setting up a new feed and speed for a tool or part. Relevant feeds and speeds would be other uses for the same tool assembly for the same or similar materials, other uses for the same cutting tool for the same or similar materials, other tool assemblies with a similar type for the same or similar materials.

\section{Query}

Role - All Users

Query for Tool Lists with the ability to limit return on at least the following information.

Part Number

Operation Number

Program number

Part Material

Target Machine

Owning Engineer for the Tool List

Specific Tool Assembly in the List

Specific Cutting Tool in the List

Specific Insert in the List

Specific Removable Shank in the List

Specific Holder in the List

Specific Extension in the List

Specific Collet in the List

For character fields, support exact matches and wildcard matches 
For numeric fields, support exact matches, minimum limits, maximum limits, and range values. For character fields with a list of values, offer the list of available values to aid the user.

\section{Reporting}

Role - All Users

Support reports that output the results from the query. The report shall include all the components that make up an entry meeting the query criteria, along with the major fields for each component. User customizable is preferred.

Support output in the following formats:

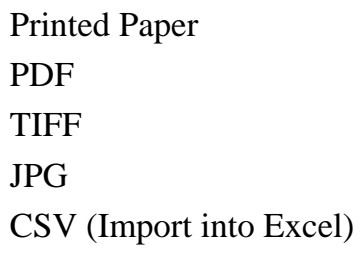

\section{Part Materials}

\section{Inserting, Updating \& Deleting}

Role - Selected Process Engineers

Allow selected Process Engineers access to maintain the tables retaining information about part materials.

Additional Information if the current Database Tables are used.

The primary database tables in the current application are MAIN_MATERIALS, MATERIAL_GROUPS, MATERIALS

\section{Desirable Requirements}

Requirements in this section represent new capabilities that would be useful to include into a new interface.

\section{Links to PeopleSoft}

PeopleSoft maintains a single field text description of all perishable cutting tool components. Additionally, PeopleSoft maintains the quantity of these perishable items that are currently available in the inventory system.

\section{PeopleSoft Fields to display in the application interface}

The values defined from PeopleSoft may be displayed directly from the PeopleSoft tables or updated once a day from PeopleSoft into the Cutting Tool Database tables.

The quantity of cutting tool, insert, spare part, or removable shank currently shown in PeopleSoft as the Stores quantity shall be made available through the Cutting Tool Database Interface. The value may be displayed directly on the Cutting Tool display screen or available with a single button click.

The PeopleSoft Item status (Active or Inactive) shall be made available through the Cutting Tool Database Interface. The value may be displayed directly on the Cutting Tool display screen or available with a single button click.

\section{Graphics}

2-D and 3-D graphics unique to a component provide an excellent tool to aid users in visualizing components and assemblies. Often, tabular data is insufficient for users to completely understand what a component looks like and if it is suitable for the intended purpose. 


\section{Internally Generated Interface}

\section{Insert, Update and Delete Graphics}

Provide a method within the interface for Cutting Tool Engineers and selected Process Engineers to setup a connection between a Cutting Tool, Insert, Removable Shank, Holder, Extension, Collet or Tool Assembly and a graphic representing the item. For some of the items, the graphic will be unique to that specific item while others will share single generic graphic of the item. An example of a specific graphic would be the drawing for a special design cutting tool. An example of a generic would be drawing of a specific type of Holder that shows how each attribute relates to the actual component. For a Tool Assembly, the intent is to provide a generic graphic for the specific Tool Assembly type.

The graphics may be TIFF, JPG or other image types that are easily generated from existing plant CAD and Graphics programs. They may be stored in an external application like Matrix or within the Cutting Tool Database itself.

\section{Display Graphics}

From each of the detail screens for Cutting Tools, Inserts, Removable Shanks, Holders, Extensions, Collets, or Tool Assemblies, provide a mechanism (ie. Button) to display the related image. No manipulation of the image would be required or allowed. Provide a method to printing the image.

\section{Commercial Off The Shelf Software}

\section{Generate Graphics}

Role - Cutting Tool Engineers and Selected Process Engineers

The system shall have a tool to create new graphics for all components contained in the system. The ability to create graphics from scratch, as well as the ability to bring up an existing graphic, modify it for a new but similar tool and then save the new graphic under a new name. The system shall come with standard graphics for all the major tool components defined in the system.

\section{Insert, Update and Delete Graphics}

Role - Cutting Tool Engineers and Selected Process Engineers

Users with the capability of inserting and deleting components shall have the ability to attach graphics to new or updated components. Components shall have the ability to attach 2-D graphics, 3-D graphics and photographs of individual components.

\section{View Graphics}

Role - All Users

When the user has the interface focus pointed to a specific component or assembly the system shall allow the user to easily view the related graphics for the component.

\section{Build Graphics for Assemblies}

\section{Role - All Users}

The system shall automatically build Tool Assembly graphics from the individual component graphics. The resulting graphics shall support 2-D representation as well as the ability to support 3-D Cad systems (See Numerical Control Support, for specific CAD Software to support) and Vericut for NC Program Generation support.

\section{Reports}

\section{Standard Reports}

Role All Users 
The system shall come with standard reports surrounding all the functional areas of the software. For Components, Standard reports shall contain detail information about the component and a graphical sketch of the component.

For Assemblies, Standard reports shall contain detail information about the Assembly as well as the list of components comprising the assembly. Graphical representations of the assembly shall also be included in the report.

\section{User Customizable}

Role - Cutting Tool Engineers and Selected Process Engineers

The system shall come with a report generator that allows selected users the ability to create new reports or to modify copies of the standard reports. When saved complete, the new reports shall be available to all users of the system. User generated reports shall have the ability to include graphics related to the items included in the report.

\section{Numerical Control Support}

Role - NC Analyst

Generation of Numerical Control (NC) programs is done utilizing Computer Aided

Manufacturing (CAM) software. We currently support (3) CAM software packages: Unigraphics, ProManufacture and MasterCam. We also have the capability to simulate the NC programs with Vericut. Creating tool data in all four systems is a manual operation today. The ability to import this information from a cutting tool management system would greatly increase productivity and program accuracy. In addition, the ability to import the final feeds and speeds from the CAM software would provide "closed loop" capability to complete the documentation of tool assembly usage.

\section{Requirements}

Export Tool Assembly definitions to CAM and Simulation systems

Rotating tool assembly (drills, reamers, end mills, etc.) 2D profile dimensions

- Holder

- Sub Attachments (if any) (extensions, collets, shell mill head, etc.)

- Cutting tool

- $\quad$ Cutting diameter

- Flute length

- Shank diameter

- Projection length

Turning tool assembly 3D profile dimensions

- Holder

- Sub Attachments (if any) (extensions, collets, boring bars, etc.)

- Cutting tool

- Insert

- $\quad$ Type (80 degree, 35 degree, etc.)

- Orientation angle

- $\quad$ Size dimensions

- Inscribed circle (diamond inserts) 
- $\quad$ Length and Width (rectangular type)

- Corner radius

- Thickness

- $\quad$ Special Profile cutters

- Solid model definition

Export Cutting Parameters for a Tool Assembly to CAM and Simulation systems

- Number of cutting edges (flutes)

- Inserts - orientation angle

- Hand of cut (left or right)

- Feed (IPM, MMPM, IPR, MMPR, or IPT) and Speed (RPM or SFM)

- Axial and Radial Depths of Cut

Import Cutting Parameters from CAM system for Tool Assembly (Specific to Tool List)

- Axial and Radial Depths of Cut

- $\quad$ Feed (IPM, MMPM, IPR, MMPR, or IPT) and Speed (RPM or SFM)

\section{Query}

Role - NC Analyst

Ability to query by:

- $\quad$ Program ID

- Tool Assembly ID

- $\quad$ Part Number

\section{Import data from Vendors}

\section{Cutting Tool Components}

Cutting tool

Holders

Accessories

\section{Cutting Data}

Feed and Speeds

Depths of Cut (radial and axial)

\section{Tool Components}

Holders

Extensions

Collets

\section{Manufacturing Support}

General desc

Provide Report generation of tool lists (set sheet and/or shopping list)

requirements 
Maintain Quantities of Machine Specific Accessories by Department requirements

Support Downloads to Tool Setters for Automatic Measurement requirements 


\section{Assembly Database Table Structure}

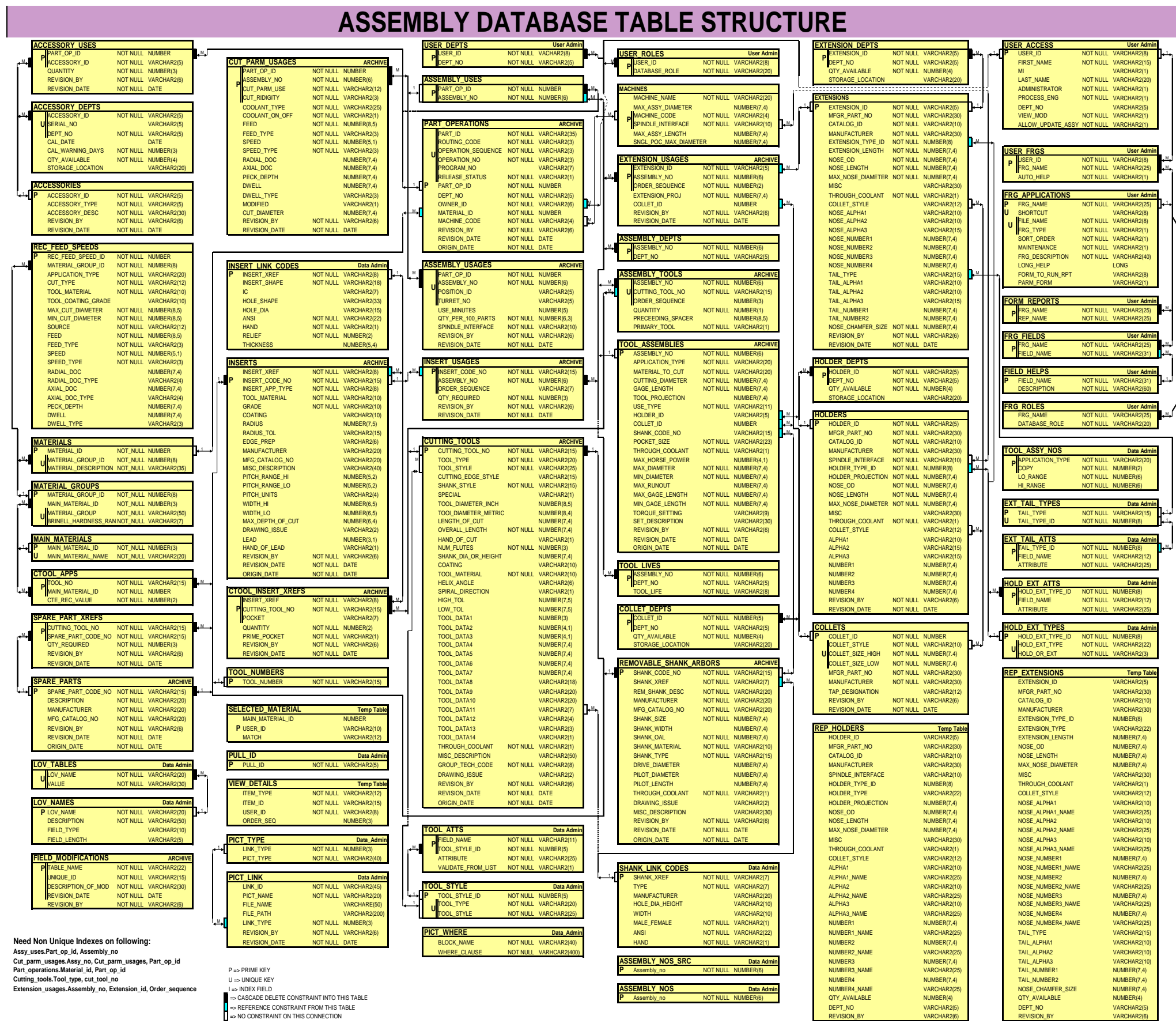




\section{Sample Database Table of Contents}

Qty of Defined Cutting Tools - 4765

Qty of Defined Inserts - 493

Qty of Defined Spare Parts - 403

Qty of Removable Shanks Defined - 47

Qty of Unique Shank Link Codes - 34

Qty of Unique Insert Link Codes - 354

Qty of Cutting Tool Styles - 98

Qty of Special Cutting Tool Attributes - 623

Qty of Holders Defined - 162

Qty Extensions Defined - 53

Qty of Collets Defined - 517

Qty of Unique Holder and Extension Types - 37

Qty of Special Holder and Extension Attributes - 96

Qty of Special Extension Left End Types - 5

Qty of Special Extension Left End Attributes - 11

Qty of Tool Assemblies Defined -1052

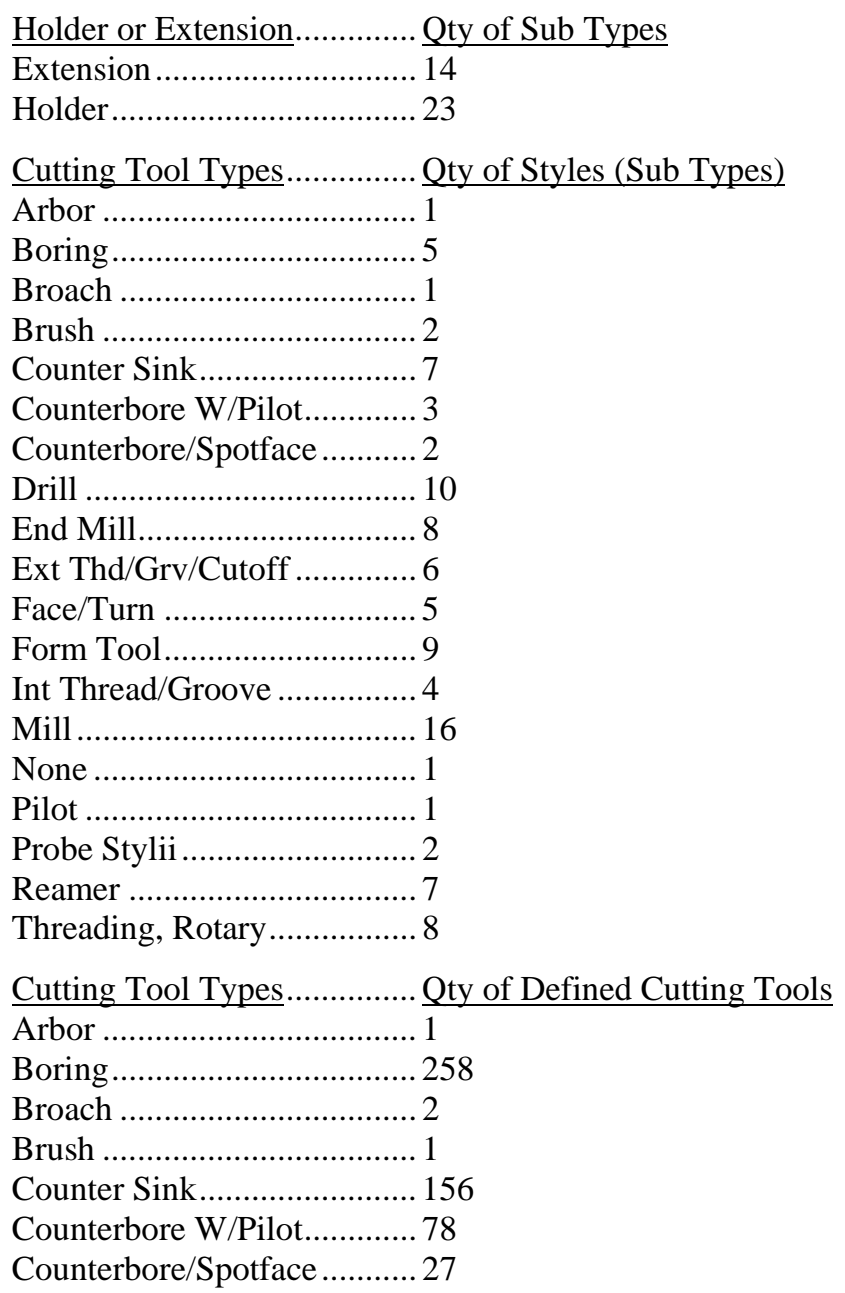




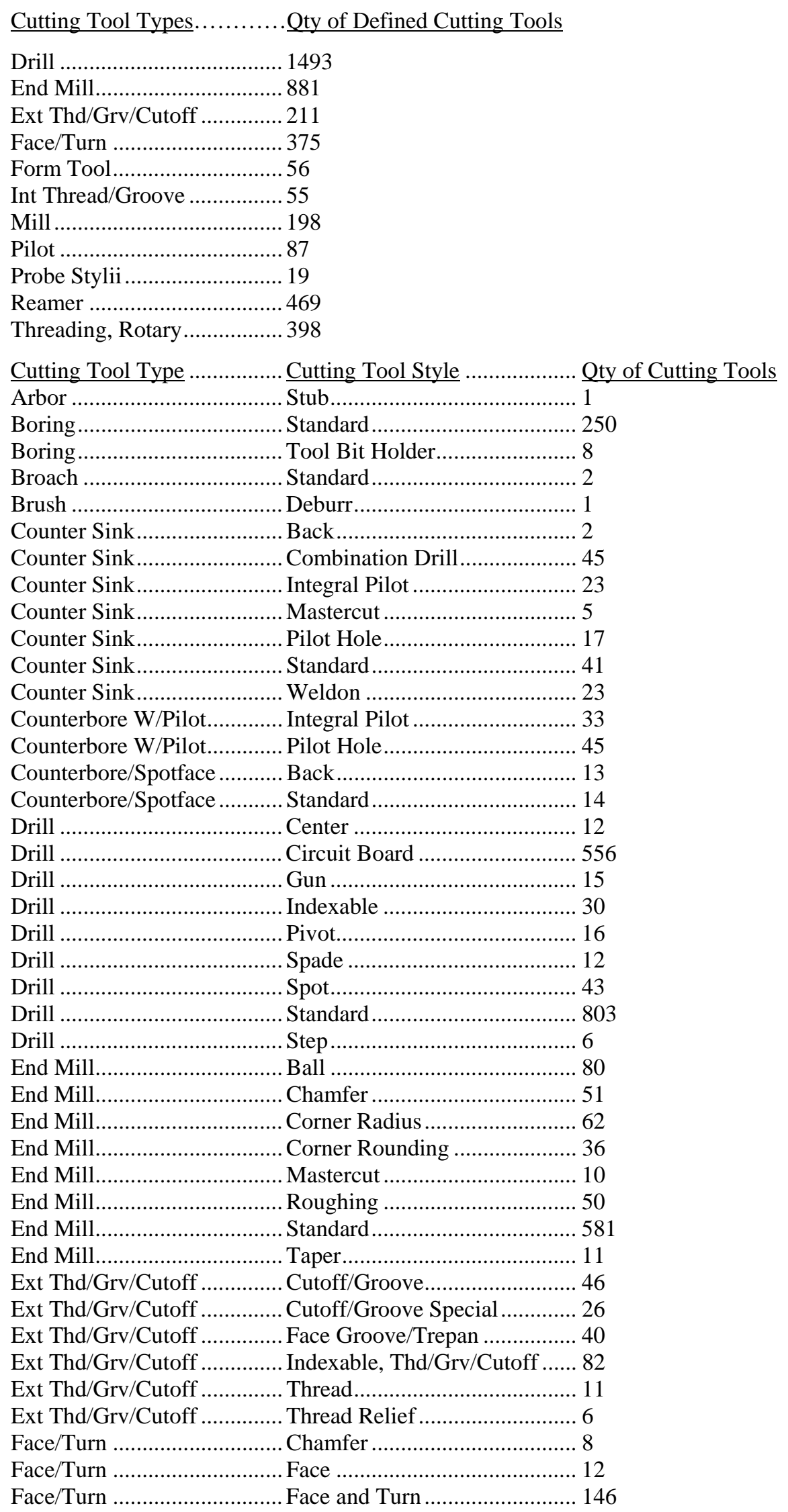




\begin{tabular}{|c|c|}
\hline \multicolumn{2}{|r|}{ Qty of Cutting Tools } \\
\hline \multicolumn{2}{|r|}{8} \\
\hline Face/Turn ........ & 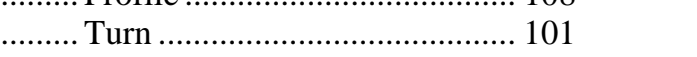 \\
\hline Form Tool.... & .. Dove Tail ............................ \\
\hline Form Tool..... & 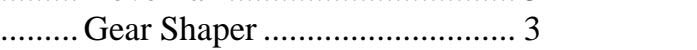 \\
\hline Form Tool............... & 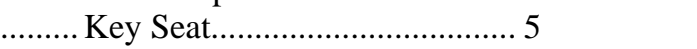 \\
\hline Form Tool................ & ........... Key Seat - Mastercut ................... 1 \\
\hline Form Tool.......... & 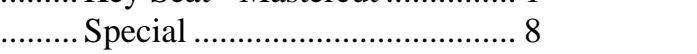 \\
\hline Form Tool............. & ... T-Slot............................................ 12 \\
\hline Form Tool............. & ..... T-Slot - Mastercut....................... 15 \\
\hline Form Tool................... & ......... Woodruff Key Seat.................... 7 \\
\hline Int Thread/Groove. & 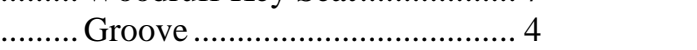 \\
\hline Int Thread/Groove. & ......... Indexable, Thd/Grv/Cutoff ...... 15 \\
\hline Int Thread/Groove . & ........ Thread...................................... 20 \\
\hline Int Thread/Groove. & .......... Thread Relief ................................ 16 \\
\hline Mill ........................... & ..... Concave Side Milling ................ 2 \\
\hline Mill ....................... & .. DA Side Milling Cutter ............. 2 \\
\hline Mill ............................ & .. Face Mill............................... 9 \\
\hline 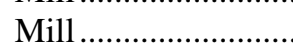 & 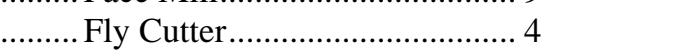 \\
\hline Mill ........................... & 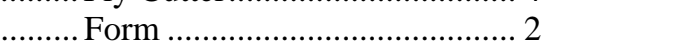 \\
\hline Mill ........................... & 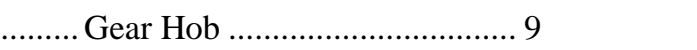 \\
\hline Mill .............. & .... Hole Saw ............................ \\
\hline Mill ....................... & ... Plain Milling Cutter ...................... 2 \\
\hline Mill ............................ & ... SA Side Milling Cutter ............. 4 \\
\hline 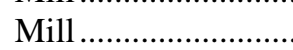 & 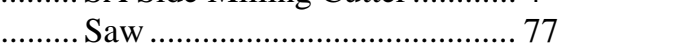 \\
\hline Mill ........................... & ....... Saw - Mastercut ............................ 5 \\
\hline 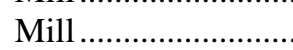 & 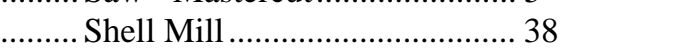 \\
\hline Mill ........................... & ....... Side Milling Cutter ...................... 11 \\
\hline Mill ......................... & ..... Side Milling Cutter Set............. 5 \\
\hline Mill .............................. & .... Thread/Groove Mill................... 1 \\
\hline Pilot ............................ & 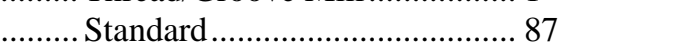 \\
\hline Probe Stylii .............. & 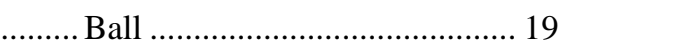 \\
\hline Reamer ..................... & 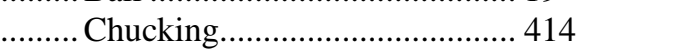 \\
\hline Reamer .................... & ......Form .................................... 21 \\
\hline 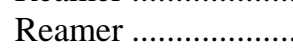 & 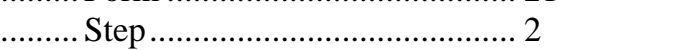 \\
\hline Reamer ...................... & ......... Stub Screw Machine.................. 11 \\
\hline Reamer ..................... & ..... Taper................................... 16 \\
\hline Reamer ............................. & .... Taper Pipe.................................... 5 \\
\hline Threading, Rotary.. & .... Cold Form Tap ........................... 21 \\
\hline Threading, Rotary... & ..... Helicoil Tap ................................ 57 \\
\hline Threading, Rotary.. & .... Oversize Helicoil Tap................. 6 \\
\hline Threading, Rotary... & ........ Pipe Тар.................................... 10 \\
\hline Threading, Rotary... & ....... Pulley Tap..................................... 2 \\
\hline Threading, Rotary... & ....... Standard Tap............................... 279 \\
\hline Threading, Rotary... & .... Thread Mill.... \\
\hline
\end{tabular}

\section{Glossary}

\footnotetext{
Administrator ------------ An individual that has responsibility to maintain fields of the database that most users only have view capability on. This would typically be a Cutting Tool Engineer or selected Process Engineers.

Assembly ---------------- Short for Tool Assembly (see Tool Assembly)

ATTS -Short for attributes
} 

An expandable or contractible metal sleeve that is generally used to hold bar stock or cutting tools.

Cut Parm Usages -------- Cutting Parameter Usages. The feeds, speeds and other cutting parameters used for a specific cutting tool.

Cutting Tool-------------- A tool that is used to remove metal, plastic or other materials using a shear cutting action.

DEPTS ------------------- Short for Departments.

Extension ----------------- A device to hold cutting tools that has an interface on the spindle end that fits into a holder or another extension but not into the machine.

EXT Commonly used as a short version of Extension.

Hold -------------------- Commonly used as a short version of Holder.

Holder------------------- A device to hold cutting tools that has an interface that mates to the machine spindle.

Indexible ----------------- A cutting tool category that describes cutting tools with replaceable cutting edges. These replaceable are generally referred to as Inserts.

Insert -------------------- A replaceable cutting edge made to be used with indexible holders. Generally made from carbide or ceramic but may be made from other materials.

Insert Link Codes-------- These are cross reference listings that allow an insert to be automatically connected with an indexible tool holder designed to hold an insert of that style. These codes are created and maintained by Cutting Tool Engineering.

IPM------------------------ Inches Per Minute

IPR ------------------------ Inches Per Revolution

IPT-------------------------- Inches Per Tooth

LOV ---------------------- List of Values. These are used for fields where the values of the field are limited to a specific group of values or common values are offered to speed entry.

Machine------------------- A device that holds a piece part and a tool assembly for the purpose of modifying the piece part. May be manually or computer controlled.

Modification Status------ Part Operation and related child records that are currently being edited or reviewed and are not available for manufacturing to use for building product.

Operation No------------- The operation number of a specific part operation. An operation is a group of activities that occur at the same time on a specific machine or in a specific location.

Operation Sequence ----- A group of operations that occur sequentially on the same machine or at the same location.

Part Operation------------ A group of activities that logically occur to a part at the same time.

Release Status ------------- Part Operation and related child records that are available to manufacturing for building product.

RPM------------------------ Revolutions Per Minute

SFM ----------------------- Surface Feet Minute

Shank Link Codes ------- These are cross reference listings that allow a removable shank to be automatically connected with a end piece designed to fit a specific shank. These codes are created and maintained by Cutting Tool Engineering.

Tool Assembly ----------- The combination of a cutting tool, extensions and a holder.

Tool Setting -------------- The process of measuring the diameter and length offsets (variation from nominal) for a built up Tool Assembly. 
Tool Style---------------- This is the name of a minor heading of a type of cutting tool. These are subtypes of Tool Types. For example, for a Tool Type of Drill, Tool Styles would include, standard (also know as twist), center, spot, and etc.

Tool Type----------------- This is the name of a major heading of a type of cutting tool. For example, these would include Drill, End Mill, and etc.

QTY ------------------- Short for quantity

User --------------------- User of the Assembly Database 


\section{Appendix B}

\section{SQL to Generate a Data File From the Existing System}

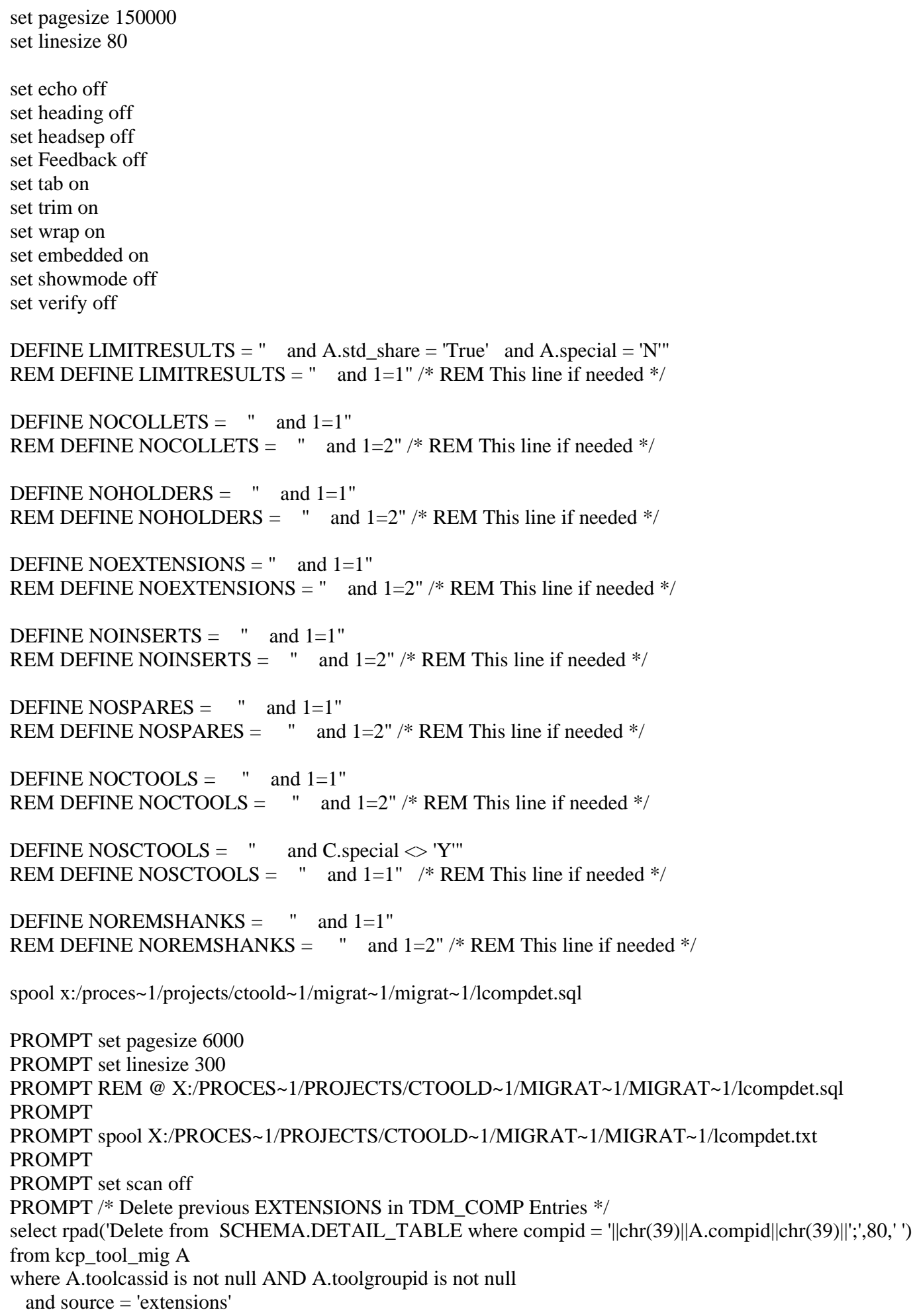




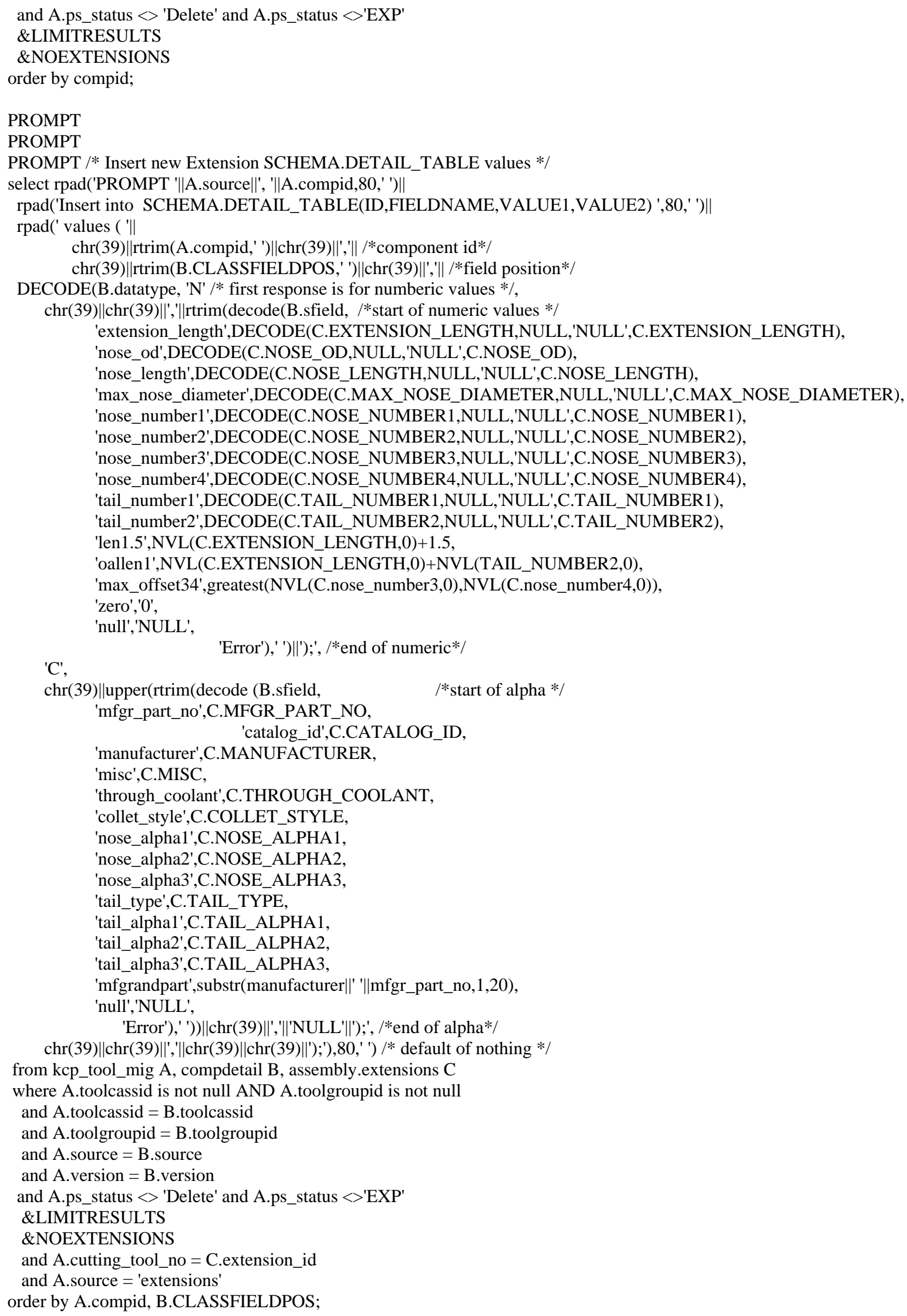




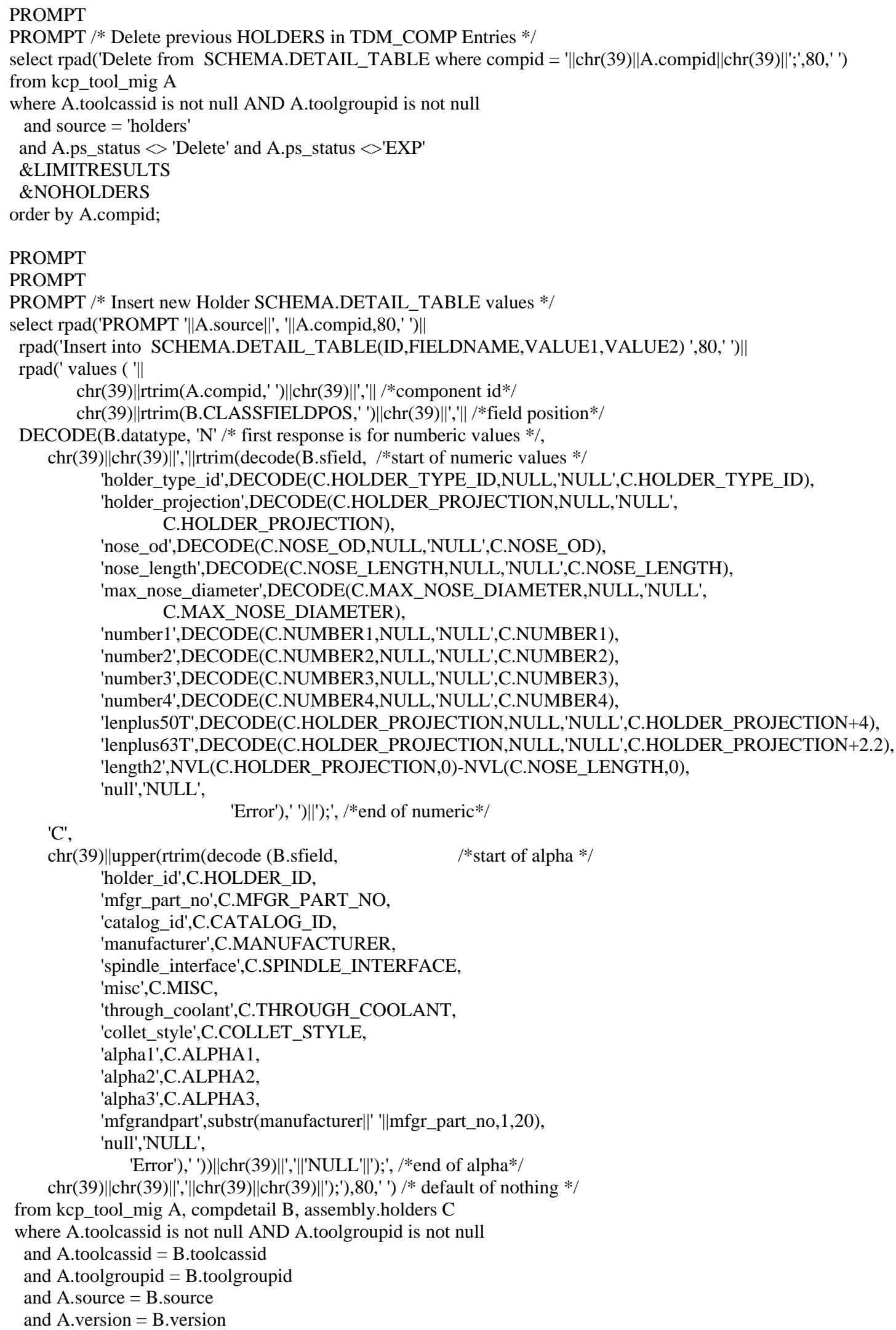




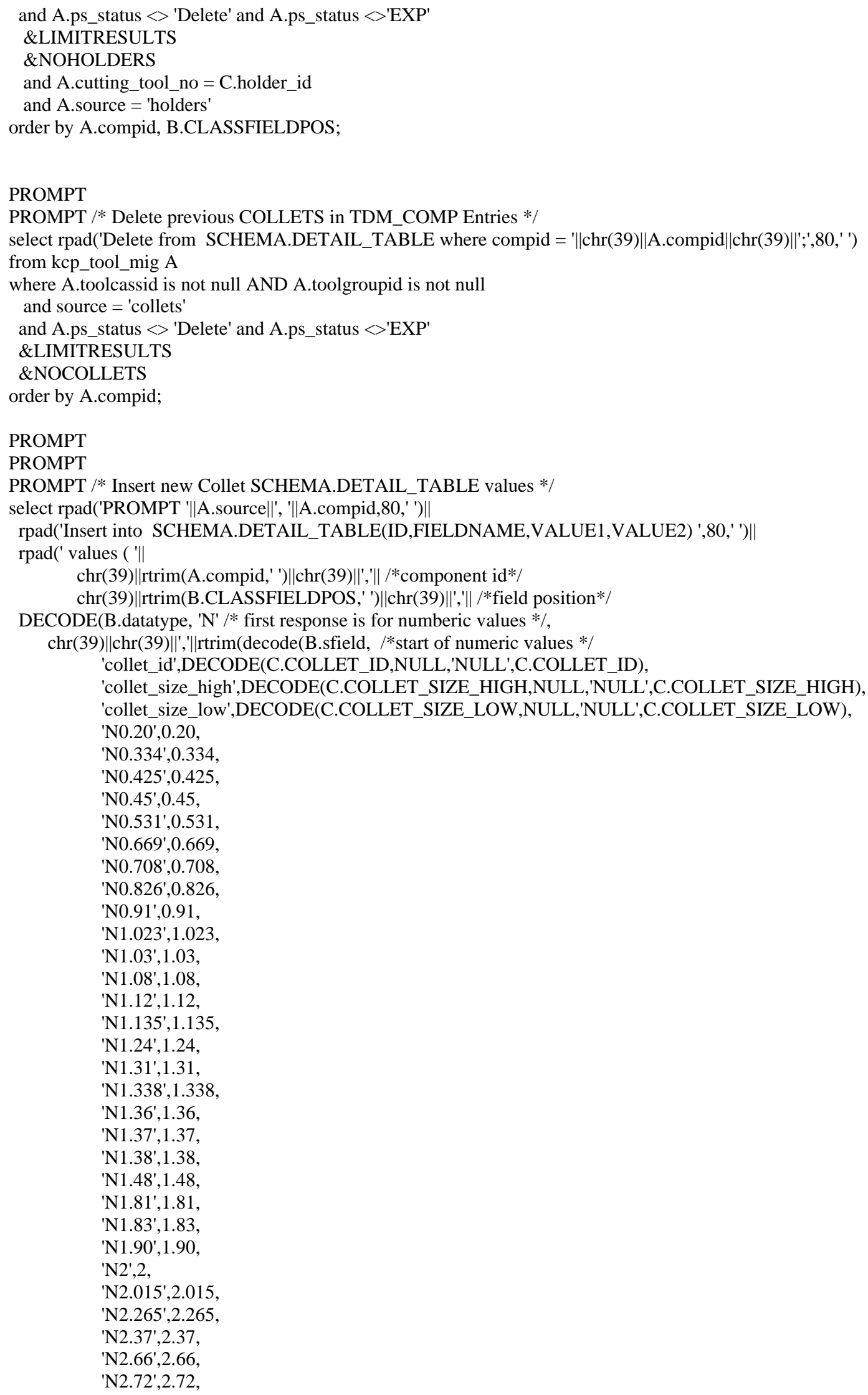




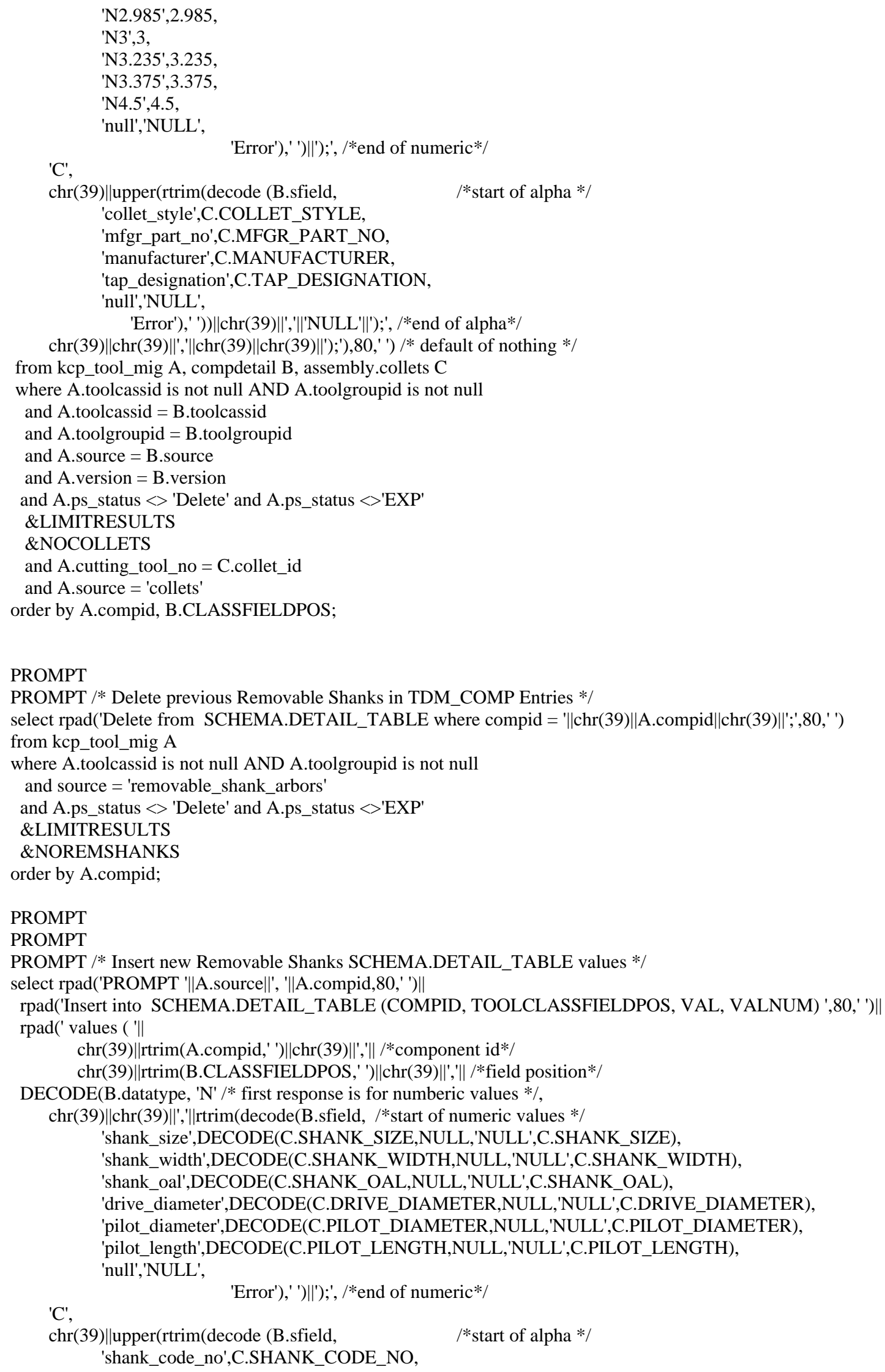


'shank_xref',C.SHANK_XREF,

'rem_shank_desc',C.REM_SHANK_DESC,

'manufacturer',C.MANUFACTURER,

'mfg_catalog_no',C.MFG_CATALOG_NO,

'shank_material',C.SHANK_MATERIAL,

'shank_type',C.SHANK_TYPE,

'through_coolant',C.THROUGH_COOLANT,

'drawing_issue',C.DRAWING_ISSUE,

'misc_description',C.MISC_DESCRIPTION,

'shank_Xref',D.SHANK_XREF,

'type',D.TYPE,

'manufacturer',D.MANUFACTURER,

'hole_dia_height',D.HOLE_DIA_HEIGHT,

'width',D.WIDTH,

'male_female',D.MALE_FEMALE,

'ansi',D.ANSI,

'hand',D.HAND,

'null','NULL',

'Error'),' '))||chr(39)||','||'NULL'||');', /*end of alpha*/

chr(39)||chr(39)||','||chr(39)||chr(39)||');'),80,' ') /* default of nothing */

from kcp_tool_mig A, compdetail B, assembly.removable_shank_arbors C, assembly.shank_link_codes D

where A.toolcassid is not null AND A.toolgroupid is not null

and A.toolcassid $=$ B.toolcassid

and A.toolgroupid = B.toolgroupid

and A.source $=$ B.source

and A.version $=$ B.version

and A.ps_status $<>$ 'Delete' and A.ps_status $<>$ 'EXP'

\&LIMITRESULTS

\&NOREMSHANKS

and A.cutting_tool_no = C.shank_code_no

and A.source = 'removable_shank_arbors'

and C.shank_xref $=$ D.shank_xref $(+)$

order by A.compid, B.CLASSFIELDPOS;

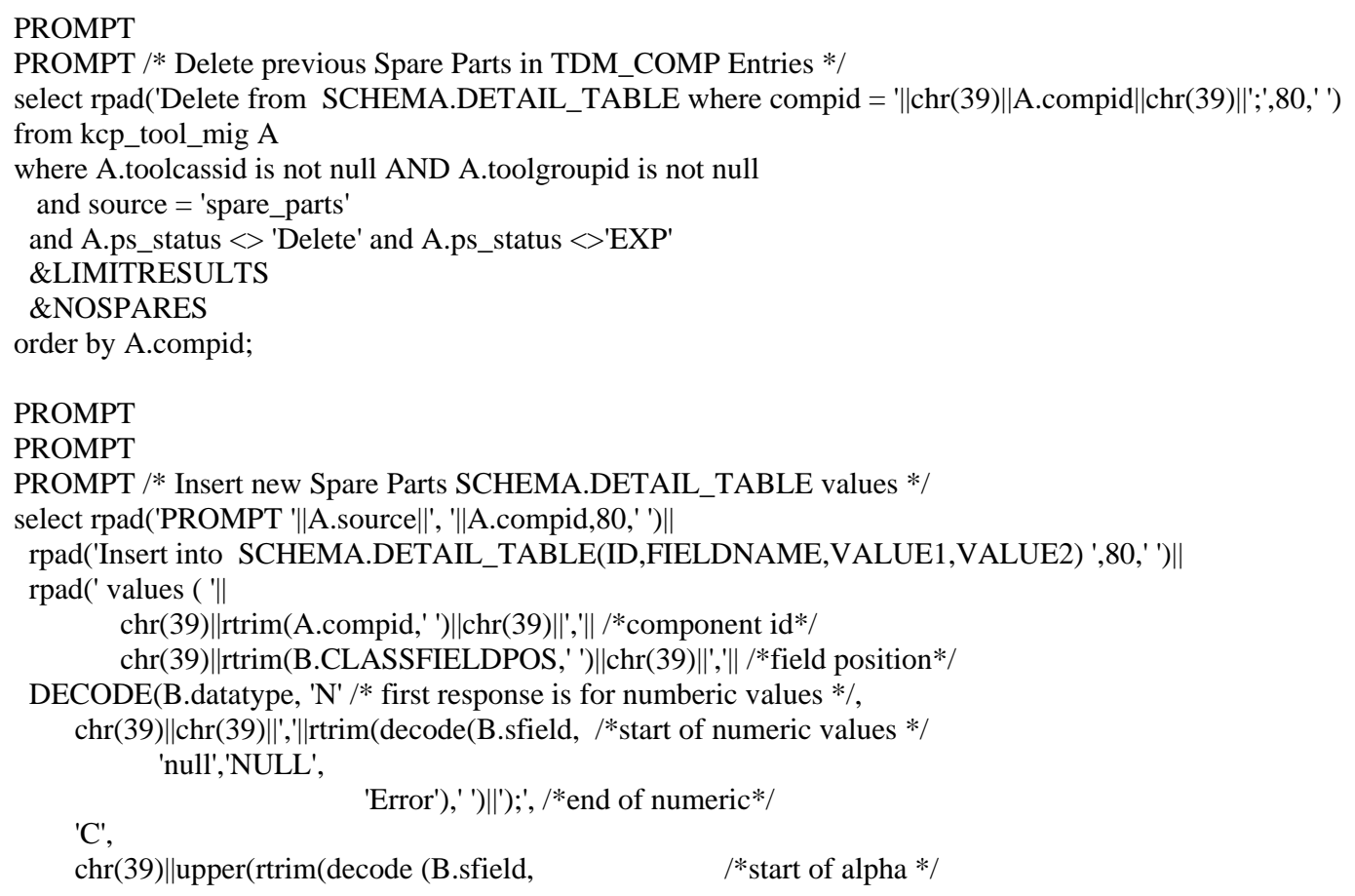


'spare_part_code_no',C.SPARE_PART_CODE_NO, 'description',INITCAP(C.DESCRIPTION),

'manufacturer',C.MANUFACTURER,

'mfg_catalog_no',C.MFG_CATALOG_NO,

'mfgrandpart',substr(C.manufacturer||' '||C.mfg_catalog_no,1,20),

'desc_type',substr(C.description||' '||C.mfg_catalog_no,1,20),

'null','NULL',

'Error'),' '))||chr(39)||',||'NULL'||');', /*end of alpha*/

$\operatorname{chr}(39)\|\operatorname{chr}(39)|| ', '|| \operatorname{chr}(39)\| \operatorname{chr}(39)|| ') ; '), 80,{ }^{\prime}$ ') /* default of nothing */

from kcp_tool_mig A, compdetail B, assembly.spare_parts C

where A.toolcassid is not null AND A.toolgroupid is not null

and A.toolcassid $=$ B.toolcassid

and A.toolgroupid = B.toolgroupid

and A.source $=$ B. source

and A.version $=$ B.version

and A.ps_status <> 'Delete' and A.ps_status <>'EXP'

\&LIMITRESULTS

\&NOSPARES

and A.cutting_tool_no $=$ C.spare_part_code_no

and A.source = 'spare_parts'

order by A.compid, B.CLASSFIELDPOS;

PROMPT

PROMPT /* Delete previous Archive Spare Parts in TDM_COMP Entries */

select rpad('Delete from SCHEMA.DETAIL_TABLE where compid = '||chr(39)||A.compid||chr(39)||';',80,' ')

from kcp_tool_mig A

where A.toolcassid is not null AND A.toolgroupid is not null

and source = 'archive_spare_parts'

and A.ps_status <> 'Delete' and A.ps_status <>'EXP'

\&LIMITRESULTS

\&NOSPARES

order by A.compid;

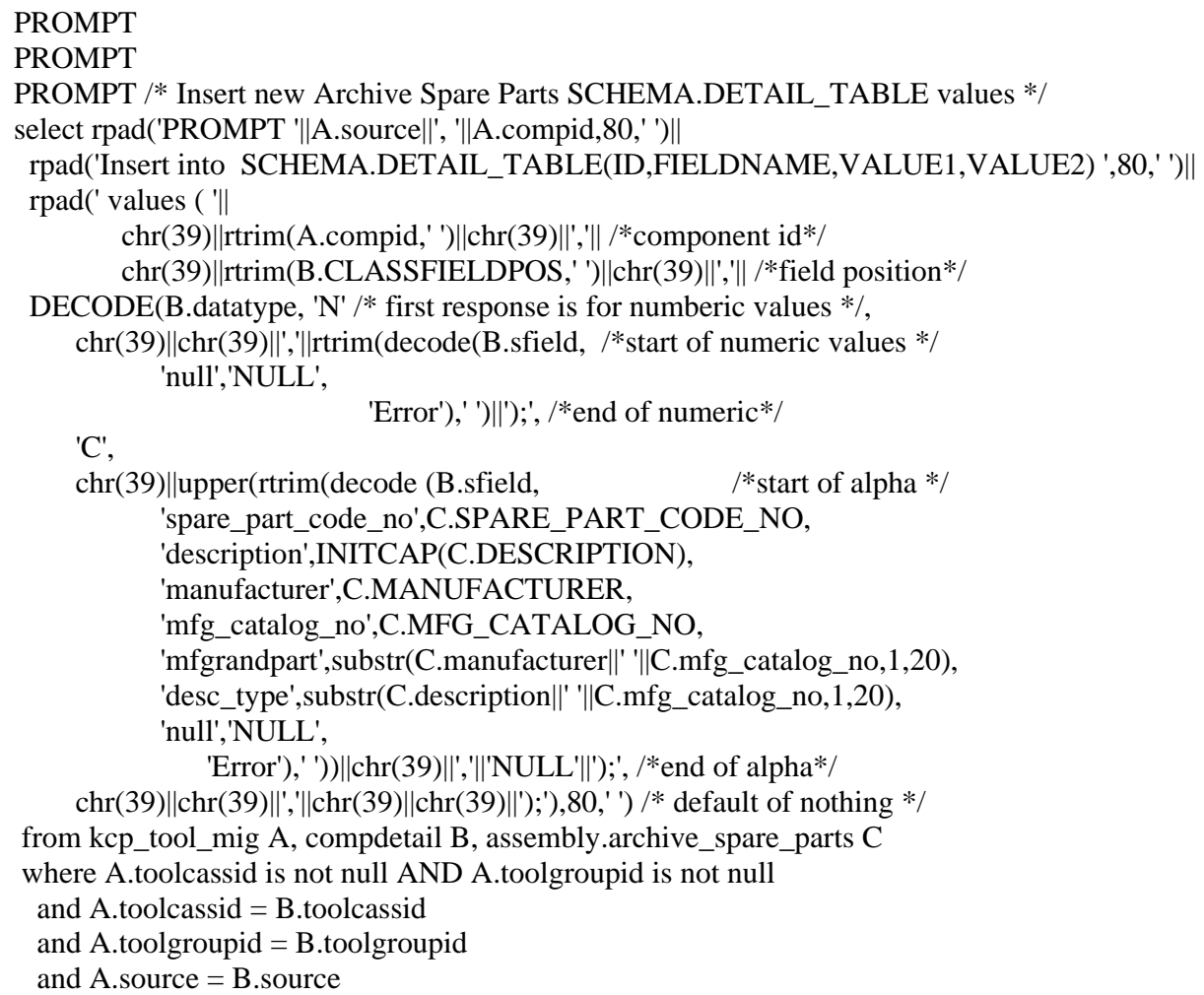


and A.version $=$ B.version

and A.ps_status <> 'Delete' and A.ps_status <>'EXP'

\&LIMITRESULTS

\&NOSPARES

and A.cutting_tool_no = C.spare_part_code_no

and A.archive_date $=$ C.archive_date

and A.source = 'archive_spare_parts'

order by A.compid, B.CLASSFIELDPOS;

\section{PROMPT}

PROMPT /* Delete previous Cutting Tools in TDM_COMP Entries */

select rpad('Delete from SCHEMA.DETAIL_TABLE where compid = '||chr(39)\|A.compid\|chr(39)||';',80,' ')

from kcp_tool_mig A, assembly.cutting_tools C

where A.toolcassid is not null AND A.toolgroupid is not null

and source $=$ 'cutting_tools'

and A.cutting_tool_no $=$ C.cutting_tool_no

and A.ps_status <> 'Delete' and A.ps_status <>'EXP'

\&NOSCTOOLS

\&NOCTOOLS

\&LIMITRESULTS

order by A.compid;

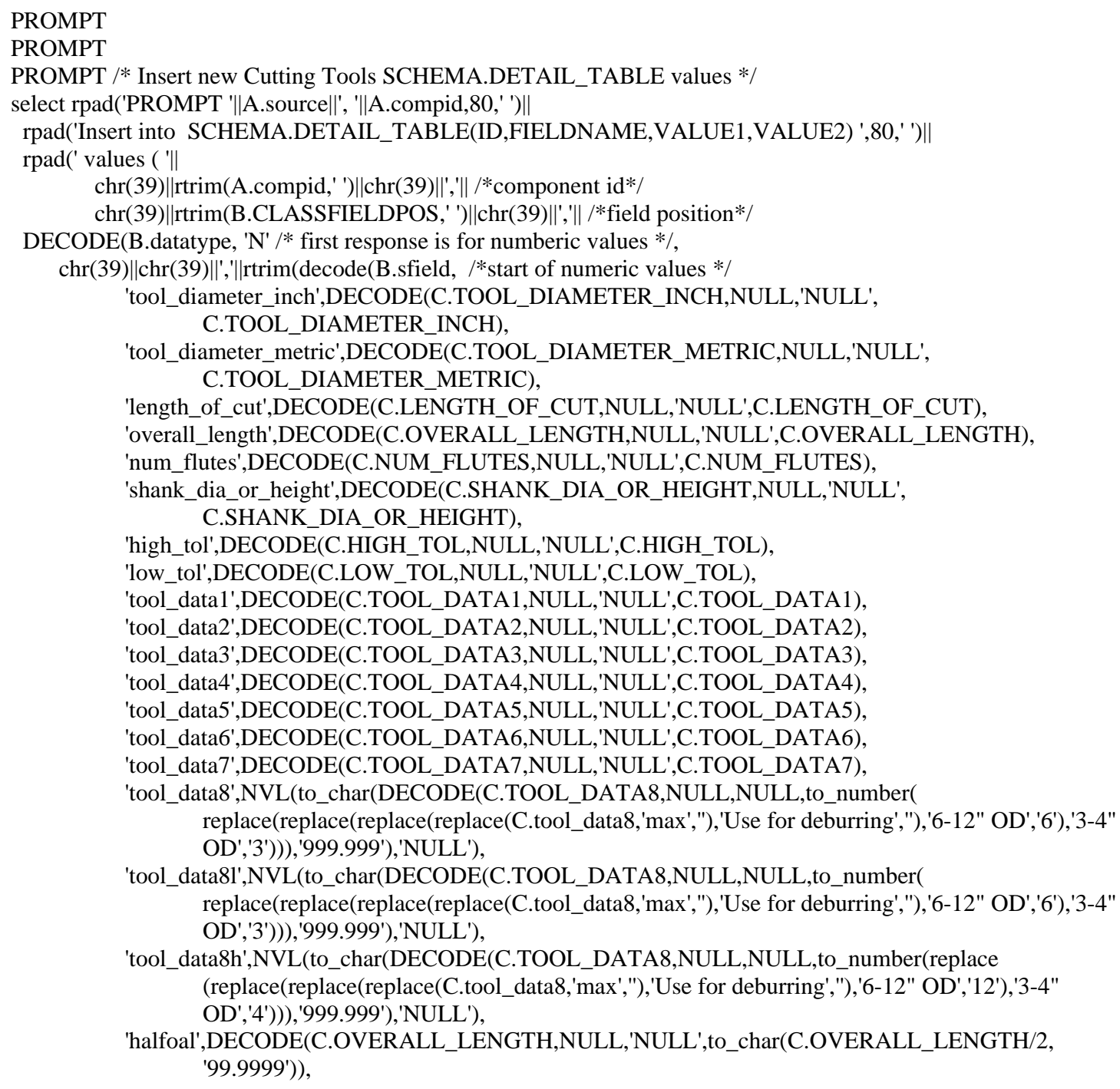


'dir_of_cut',DECODE(C.HAND_OF_CUT,NULL,'NULL','R',0,'L',1,'NULL'),

'thru_coolant',DECODE(C.THROUGH_COOLANT,NULL,'NULL','Y',1,'N',1,'NULL'),

'ang118','118',

'ang180','180',

'cdtiplen118',DECODE(C.TOOL_DATA5,NULL,'NULL',to_char(C.TOOL_DATA5/3.328,'9999.999')),

'cdtiplen2',DECODE(least(C.TOOL_DATA6/*,C.TOOL_DATA5*/),NULL,'NULL', to_char(C.TOOL_DATA6/*+C.TOOL_DATA5/3.328*/,'9999.999')),

'cdtiplen1',DECODE(LEAST(C.TOOL_DIAMETER_INCH,C.TOOL_DATA4, C.TOOL_DATA6,C.TOOL_DATA5),NULL,'NULL',to_char((C.TOOL_DIAMETER_INCHC.TOOL_DATA6)/(2*TAN(C.TOOL_DATA4/(2*57.296) $)+$ +C.TOOL_DATA6/*+C.TOOL_DATA5/3.328 */,'9999.999')),

'cdtiplen_1',DECODE(LEAST(C.TOOL_DATA5,C.TOOL_DATA4),NULL,'NULL', to_char(C.TOOL_DATA5/(2*TAN(C.TOOL_DATA4/(2*57.296))),'9999.999')),

'cdtiplen_2',DECODE(LEAST(C.TOOL_DATA6/*,C.TOOL_DATA5, C.TOOL_DATA4*/),NULL,'NULL',to_char(C.TOOL_DATA6/*+(C.TOOL_DATA5/(2*TAN(C.TOOL_D ATA4/(2*57.296))))*/,'9999.999')),

'cdtiplen_3',DECODE(LEAST(C.TOOL_DIAMETER_INCH,C.TOOL_DATA7, C.TOOL_DATA6,C.TOOL_DATA5),NULL,'NULL',to_char((C.TOOL_DIAMETER_INCHC.TOOL_DATA5)/(2*TAN(DECODE(C.TOOL_DATA7,null,180,0,180,C.TOOL_DATA7)/(2*57.296) ))+ C.TOOL_DATA6,'9999.999')),

'dhelixangle',DECODE(C.HELIX_ANGLE,NULL,'NULL','NotApp',0,'Nutral',0,'Medium', '35','Low','20','High','50','Hi Pos','55','NULL'),

'dtiplen',DECODE(LEAST(C.TOOL_DIAMETER_INCH,C.TOOL_DATA4),NULL, 'NULL',to_char(C.TOOL_DIAMETER_INCH/(2*tan(C.TOOL_DATA4/(2*57.296))),'9999.999')),

'maxdia',NVL(to_char(GREATEST(C.TOOL_DIAMETER_INCH,C.SHANK_DIA_OR_HEIGHT), '9999.999'),'NULL'),

'halftool_data4',DECODE(C.TOOL_DATA4,NULL,'NULL',to_char(C.TOOL_DATA4/2,'999.99')),

'halftool_data3',DECODE(C.TOOL_DATA3,NULL,'NULL',to_char(C.TOOL_DATA3/2,'999.99')),

'gagelenlong',DECODE(LEAST(C.OVERALL_LENGTH,C.SHANK_DIA_OR_HEIGHT), NULL,'NULL',TO_CHAR(C.OVERALL_LENGTH-3*C.SHANK_DIA_OR_HEIGHT,'9999.999')),

'gagelenshort',DECODE(LEAST(C.LENGTH_OF_CUT,C.SHANK_DIA_OR_HEIGHT), NULL,'NULL',TO_CHAR(C.LENGTH_OF_CUT+3*C.SHANK_DIA_OR_HEIGHT,'9999.999')),

'reamangle',DECODE(C.TOOL_DATA2,NULL,'90',to_char(C.TOOL_DATA2,'999.99')),

'reamanglex2',DECODE(C.TOOL_DATA4,NULL,'90',to_char(C.TOOL_DATA4*2,'999.99')),

'tap_point',DECODE(C.TOOL_DATA8,NULL,'NULL','BOTTOM', to_char(DECODE(C.TOOL_DATA12,'MMPT',C.TOOL_DATA4/25.4,1/C.tool_data4),'999.999'),'PLUG',to _char(DECODE(C.TOOL_DATA12,'MMPT',5*C.TOOL_DATA4/25.4,5/C.tool_data4),'999.999'),'MODIFI ED

BOTTOM',to_char(DECODE(C.TOOL_DATA12,'MMPT',2.5*C.TOOL_DATA4/25.4,2.5/C.tool_data4),'99 9.999'),'SPIRAL

PLUG',to_char(DECODE(C.TOOL_DATA12,'MMPT',5*C.TOOL_DATA4/25.4,5/C.tool_data4),'999.999'))

'length_of_cut/2',DECODE(C.length_of_cut,NULL,'NULL',to_char(C.length_of_cut/2,'0.999')),

'chamfend',DECODE(LEAST(C.TOOL_DATA4,C.tool_diameter_inch),NULL,'NULL', to_char(round((C.tool_diameter_inch-

NVL(C.TOOL_DATA5,0))/(2*TAN(C.TOOL_DATA4/(2*57.296))),3),'9999.999')),

'chamfend1',DECODE(LEAST(C.TOOL_DATA4,C.tool_diameter_inch),NULL,'NULL', least(C.length_of_cut,to_char(round((C.tool_diameter_inch-

NVL(C.TOOL_DATA5,0))/(2*TAN(C.TOOL_DATA4/(2*57.296))),3),'9999.999'))),

'half_tool_diameter',DECODE(C.TOOL_DIAMETER_INCH,NULL,'NULL', round(C.TOOL_DIAMETER_INCH/2,4)),

'angleend',DECODE(LEAST(C.TOOL_DATA2,C.tool_diameter_inch),NULL,'NULL', to_char(round((C.tool_diameter_inch-

NVL(C.TOOL_DATA440))/(2*TAN(C.TOOL_DATA2/(57.296))),3),'9999.999')),

'2xtool_data2',DECODE(C.TOOL_DATA2,NULL,'NULL',round(2*C.TOOL_DATA2,4)),

'2xtool_data5',DECODE(C.TOOL_DATA5,NULL,'NULL',round(2*C.TOOL_DATA5,4)),

'doveangend',DECODE(LEAST(C.TOOL_DATA7,C.TOOL_DATA4,

C.tool_diameter_inch),NULL,'NULL',to_char(round((C.tool_diameter_inch-

2*NVL(C.TOOL_DATA7,0)*TAN(C.TOOL_DATA4/(57.296))),3),'9999.999')),

'gagelen2',DECODE(LEAST(C.OVERALL_LENGTH,C.SHANK_DIA_OR_HEIGHT, C.tool_data4),NULL,'NULL',TO_CHAR(C.OVERALL_LENGTH-3*C.SHANK_DIA_OR_HEIGHTC.tool_data4,'9999.999')), 


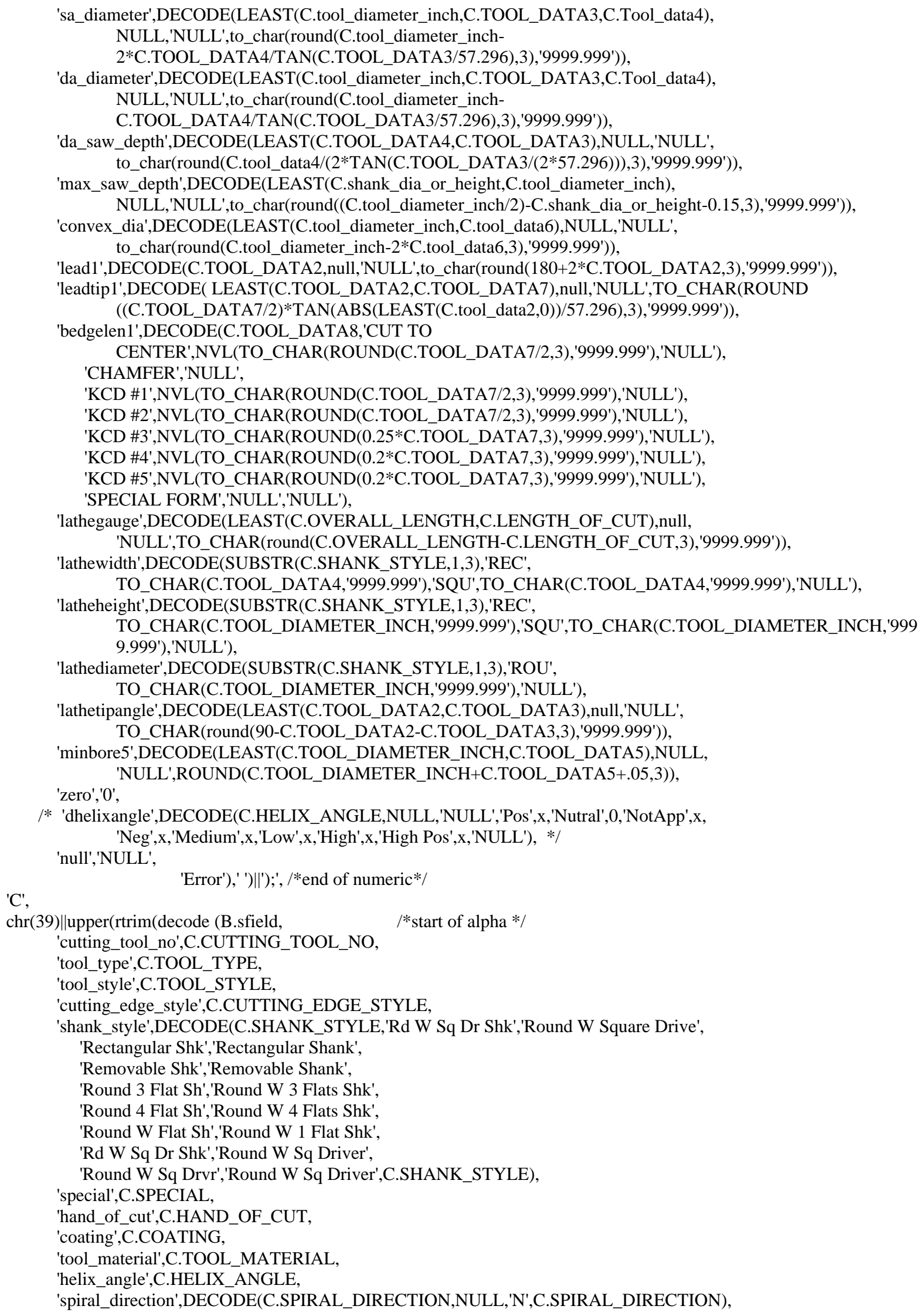


'tool_data8',C.TOOL_DATA8,

'tool_data9',C.TOOL_DATA9,

'tool_data10',C.TOOL_DATA10,

'tool_data11',C.TOOL_DATA11,

'tool_data12',C.TOOL_DATA12,

'tool_data13',C.TOOL_DATA13,

'tool_data14',C.TOOL_DATA14,

'through_coolant',DECODE(C.THROUGH_COOLANT,NULL,'N',C.THROUGH_COOLANT),

'misc_description',C.MISC_DESCRIPTION,

'group_tech_code',C.GROUP_TECH_CODE,

'drawing_issue',C.DRAWING_ISSUE,

'shank_xref',D.SHANK_XREF,

'type',D.TYPE,

'manufacturer',D.MANUFACTURER,

'hole_dia_height',D.HOLE_DIA_HEIGHT,

'width',D.WIDTH,

'male_female',D.MALE_FEMALE,

'ansi',D.ANSI,

'hand',D.HAND,

'text_pilot','INTEGRAL CUTTING PILOT',

'seeprint',DECODE(C.MISC_DESCRIPTION,NULL,'SEE PRINT',C.MISC_DESCRIPTION),

'tap_callout',REPLACE(C.tool_data9||'-'||to_char(C.tool_data4,'99990'),' ',")||' '||C.tool_data11,

'tap_callouthc',REPLACE(C.tool_data9||'-'||to_char(C.tool_data4,'99990'), ' ',")||' '||C.tool_data11||' HC',

'tap_calloutohc',REPLACE(C.tool_data9||'-'||to_char(C.tool_data4,'99990'),' ',")||' '||C.tool_data11||' OHC',

'hook',DECODE(LEAST(C.TOOL_DATA1,C.TOOL_DATA5),NULL,'NULL','HOOK

'||REPLACE(to_char(C.tool_data1,'99990')||'-'||to_char(C.tool_data5,'90'),' ',")),

'threadmilldes',REPLACE(C.tool_data8||'-'||to_char(C.tool_data4,'99990')||'-'||to_char(C.tool_data6,'99990'),' ',")||' '||C.tool_data12,

'pitchrange',REPLACE(to_char(C.tool_data4,'99990')||'-'||to_char(C.tool_data6,'99990')||C.Tool_data12,' ',"), 'pitchrange2',REPLACE(to_char(C.tool_data4,'99990')||'-'||to_char(C.tool_data3,'99990')||C.Tool_data12,' ',"), 'mastercut','MASTERCUT',

'set_info','MATCHED CUTTER SET',

'null','NULL',

'Error'),' '))||chr(39)||',||'NULL'||');', /*end of alpha*/

chr(39)\|chr(39)||','||chr(39)||chr(39)||');'),80,' ') /* default of nothing */

from kcp_tool_mig A, compdetail B, assembly.cutting_tools C,

assembly.shank_link_codes D

where A.toolcassid is not null AND A.toolgroupid is not null

and A.toolcassid $=$ B.toolcassid

and A.toolgroupid = B.toolgroupid

and A.source $=$ B.source

and A.version $=$ B.version

and A.ps_status $<>$ 'Delete' and A.ps_status $<>$ 'EXP'

\&LIMITRESULTS

\&NOCTOOLS

\&NOSCTOOLS

and A.cutting_tool_no = C.cutting_tool_no

and A.source = 'cutting_tools'

and C.tool_data $11=$ D.shank_xref $(+)$

order by A.compid, B.CLASSFIELDPOS;

\section{PROMPT}

PROMPT /* Delete previous Archive Cutting Tools in TDM_COMP Entries */

select rpad('Delete from SCHEMA.DETAIL_TABLE where compid = '||chr(39)\|A.compid||chr(39)||';',80,' ')

from kcp_tool_mig A, assembly.archive_cutting_tools C

where A.toolcassid is not null AND A.toolgroupid is not null

and source = 'archive_cutting_tools'

and A.cutting_tool_no = C.cutting_tool_no

and A.archive_date $=$ C.archive_date

and A.ps_status $<>$ 'Delete' and A.ps_status $<>$ 'EXP' 


\section{\&NOSCTOOLS \\ \&NOCTOOLS \\ \&LIMITRESULTS \\ order by A.compid;}

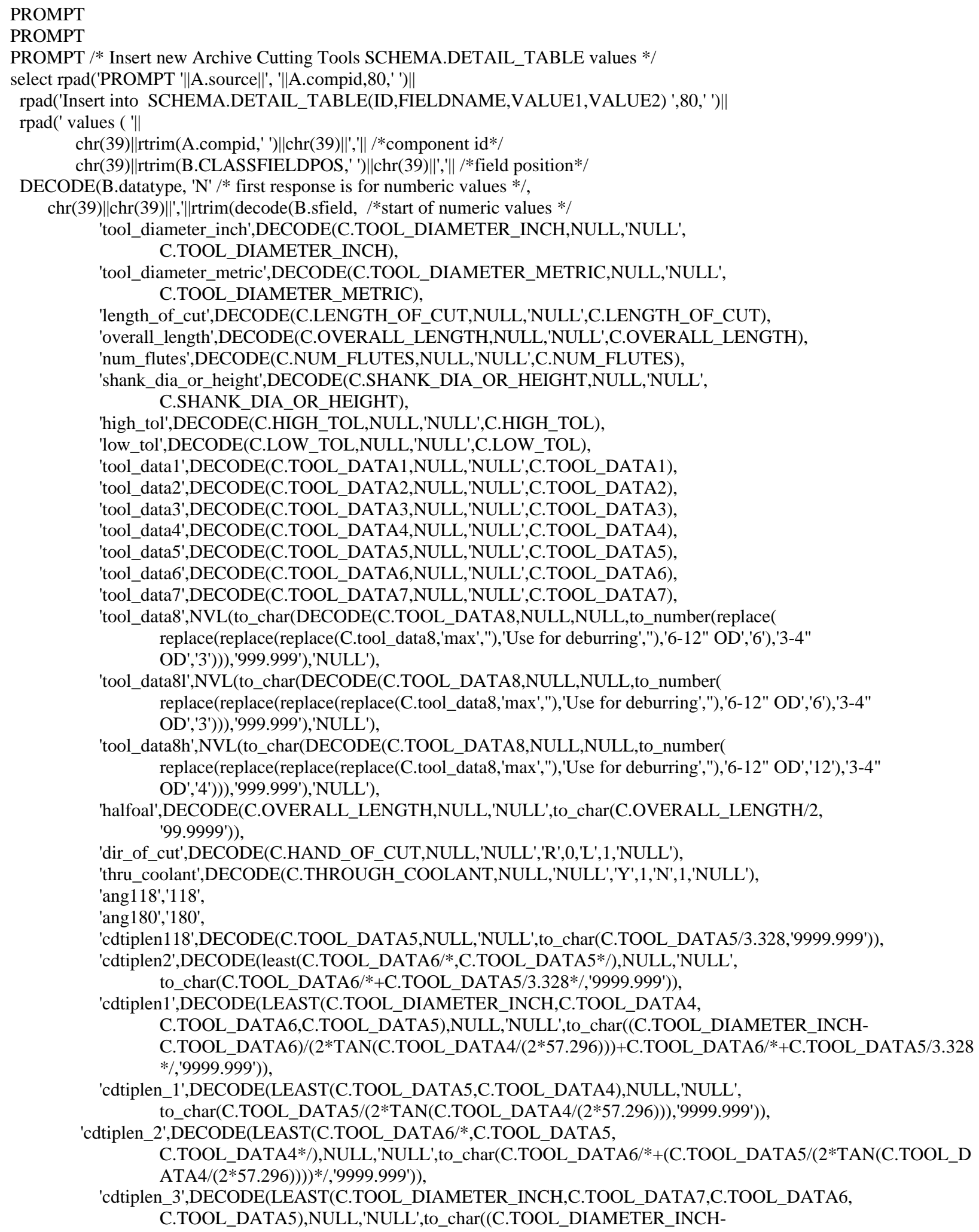


C.TOOL_DATA5)/(2*TAN(DECODE(C.TOOL_DATA7,null,180,0,180,C.TOOL_DATA7)/(2*57.296)))+ C.TOOL_DATA6,'9999.999')),

'dhelixangle',DECODE(C.HELIX_ANGLE,NULL,'NULL','NotApp',0,'Nutral',0,'Medium',' 35','Low','20','High','50','Hi Pos','55','NULL'),

'dtiplen',DECODE(LEAST(C.TOOL_DIAMETER_INCH,C.TOOL_DATA4),NULL,'NULL', to_char(C.TOOL_DIAMETER_INCH/(2*tan(C.TOOL_DATA4/(2*57.296))),'9999.999')),

'maxdia',NVL(to_char(GREATEST(C.TOOL_DIAMETER_INCH,C.SHANK_DIA_OR_HEIGHT), '9999.999'),'NULL'),

'halftool_data4',DECODE(C.TOOL_DATA4,NULL,'NULL',to_char(C.TOOL_DATA4/2,'999.99')),

'halftool_data3',DECODE(C.TOOL_DATA3,NULL,'NULL',to_char(C.TOOL_DATA3/2,'999.99')),

'gagelenlong',DECODE(LEAST(C.OVERALL_LENGTH,C.SHANK_DIA_OR_HEIGHT), NULL,'NULL',TO_CHAR(C.OVERALL_LENGTH-3*C.SHANK_DIA_OR_HEIGHT,'9999.999')),

'gagelenshort',DECODE(LEAST(C.LENGTH_OF_CUT,C.SHANK_DIA_OR_HEIGHT), NULL,'NULL',TO_CHAR(C.LENGTH_OF_CUT+3*C.SHANK_DIA_OR_HEIGHT,'9999.999')),

'reamangle',DECODE(C.TOOL_DATA2,NULL,'90',to_char(C.TOOL_DATA2,'999.99')),

'reamanglex2',DECODE(C.TOOL_DATA4,NULL,'90',to_char(C.TOOL_DATA4*2,'999.99')),

'tap_point',DECODE(C.TOOL_DATA8,NULL,'NULL','BOTTOM',

to_char(DECODE(C.TOOL_DATA12,'MMPT',C.TOOL_DATA4/25.4,1/C.tool_data4), '999.999'),'PLUG',to_char(DECODE(C.TOOL_DATA12,'MMPT',5*

C.TOOL_DATA4/25.4,5/C.tool_data4),'999.999'),'MODIFIED

BOTTOM',to_char(DECODE(C.TOOL_DATA12,'MMPT',2.5*C.TOOL_DATA4/25.4, 2.5/C.tool_data4),'999.999'),'SPIRAL

PLUG',to_char(DECODE(C.TOOL_DATA12,'MMPT',5*C.TOOL_DATA4/25.4,

5/C.tool_data4),'999.999'))

'length_of_cut/2',DECODE(C.length_of_cut,NULL,'NULL',to_char(C.length_of_cut/2,'0.999')),

'chamfend',DECODE(LEAST(C.TOOL_DATA4,C.tool_diameter_inch),NULL, 'NULL',to_char(round((C.tool_diameter_inchNVL(C.TOOL_DATA5,0))/(2*TAN(C.TOOL_DATA4/(2*57.296))),3),'9999.999')),

'chamfend1',DECODE(LEAST(C.TOOL_DATA4,C.tool_diameter_inch),NULL, 'NULL',least(C.length_of_cut,to_char(round((C.tool_diameter_inchNVL(C.TOOL_DATA5,0))/(2*TAN(C.TOOL_DATA4/(2*57.296))),3),'9999.999'))),

'half_tool_diameter',DECODE(C.TOOL_DIAMETER_INCH,NULL,'NULL', round(C.TOOL_DIAMETER_INCH/2,4)),

'angleend',DECODE(LEAST(C.TOOL_DATA2,C.tool_diameter_inch),NULL, 'NULL',to_char(round((C.tool_diameter_inchNVL(C.TOOL_DATA4,0))/(2*TAN(C.TOOL_DATA2/(57.296))),3),'9999.999')),

'2xtool_data2',DECODE(C.TOOL_DATA2,NULL,'NULL',round(2*C.TOOL_DATA2,4)),

'2xtool_data5',DECODE(C.TOOL_DATA5,NULL,'NULL',round(2*C.TOOL_DATA5,4)),

'doveangend',DECODE(LEAST(C.TOOL_DATA7,C.TOOL_DATA4,

C.tool_diameter_inch),NULL,'NULL',to_char(round((C.tool_diameter_inch-

2*NVL(C.TOOL_DATA7,0)*TAN(C.TOOL_DATA4/(57.296))),3),'9999.999')),

'gagelen2',DECODE(LEAST(C.OVERALL_LENGTH,C.SHANK_DIA_OR_HEIGHT, C.tool_data4),NULL,'NULL',TO_CHAR(C.OVERALL_LENGTH-3*C.SHANK_DIA_OR_HEIGHTC.tool_data4,'9999.999')),

'sa_diameter',DECODE(LEAST(C.tool_diameter_inch,C.TOOL_DATA3,C.Tool_data4), NULL,'NULL',to_char(round(C.tool_diameter_inch-

2*C.TOOL_DATA4/TAN(C.TOOL_DATA3/57.296),3),'9999.999')),

'da_diameter',DECODE(LEAST(C.tool_diameter_inch,C.TOOL_DATA3,C.Tool_data4), NULL,'NULL',to_char(round(C.tool_diameter_inch-

C.TOOL_DATA4/TAN(C.TOOL_DATA3/57.296),3),'9999.999')),

'da_saw_depth',DECODE(LEAST(C.TOOL_DATA4,C.TOOL_DATA3),NULL, 'NULL',to_char(round(C.tool_data4/(2*TAN(C.TOOL_DATA3/(2*57.296))),3), '9999.999')),

'max_saw_depth',DECODE(LEAST(C.shank_dia_or_height,C.tool_diameter_inch), NULL,'NULL',to_char(round((C.tool_diameter_inch/2)-C.shank_dia_or_height-0.15,3),'9999.999')),

'convex_dia',DECODE(LEAST(C.tool_diameter_inch,C.tool_data6),NULL, 'NULL',to_char(round(C.tool_diameter_inch-2*C.tool_data6,3),'9999.999')),

'lead1',DECODE(C.TOOL_DATA2,null,'NULL',to_char(round(180+2*C.TOOL_DATA2, 3),'9999.999')),

'leadtip1',DECODE( LEAST(C.TOOL_DATA2,C.TOOL_DATA7),null,'NULL', TO_CHAR(ROUND ((C.TOOL_DATA7/2)*TAN(ABS(LEAST(C.tool_data2,0))/57.296),3),'9999.999')), 


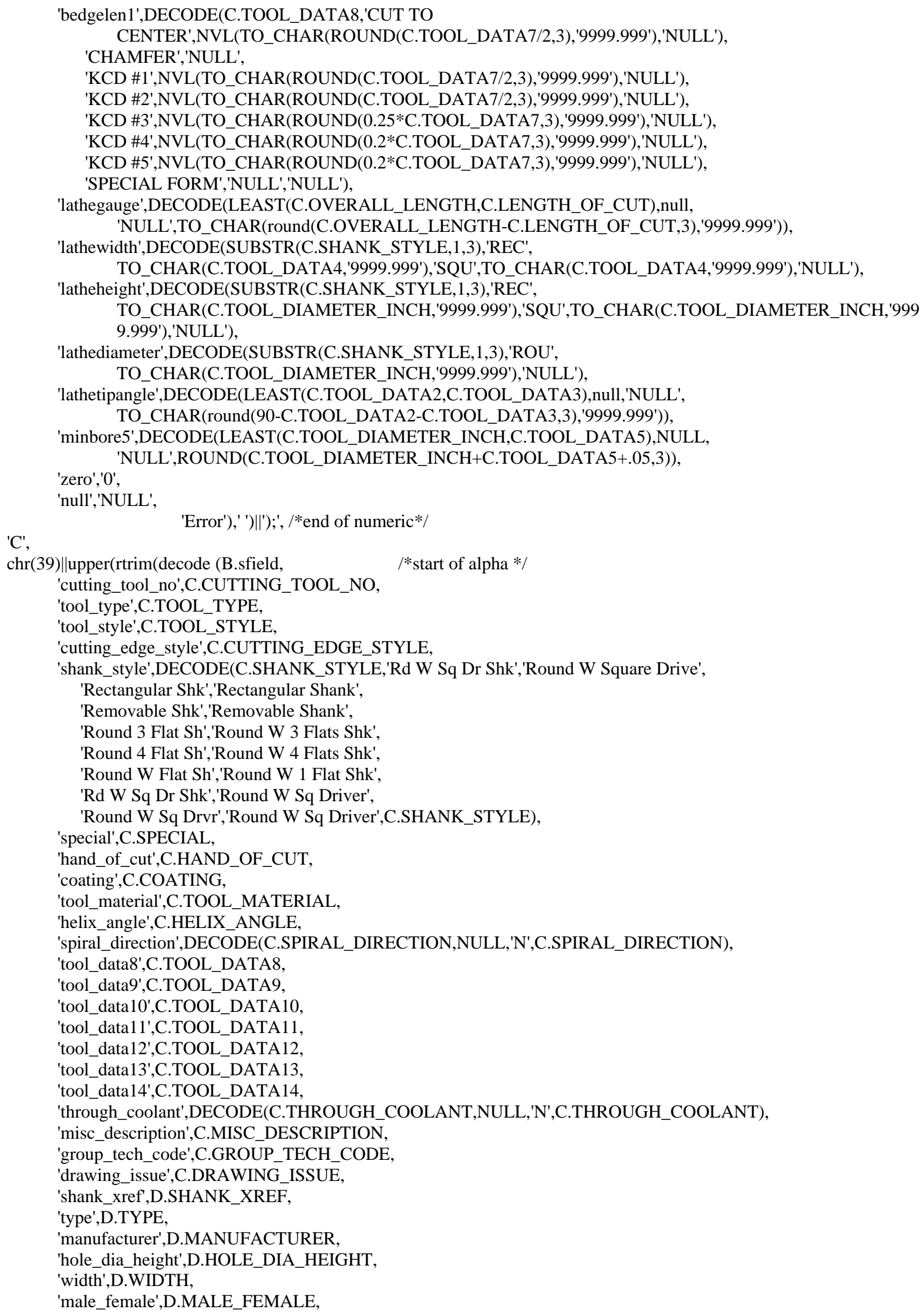


'ansi',D.ANSI,

'hand',D.HAND,

'text_pilot','INTEGRAL CUTTING PILOT',

'seeprint',DECODE(C.MISC_DESCRIPTION,NULL,'SEE PRINT',C.MISC_DESCRIPTION),

'tap_callout',REPLACE(C.tool_data9||'-'||to_char(C.tool_data4,'99990'),' ',")||' '||C.tool_data11,

'tap_callouthc',REPLACE(C.tool_data9||'-'||to_char(C.tool_data4,'99990'),' ',")||' '||C.tool_data11||' HC',

'tap_calloutohc',REPLACE(C.tool_data9||'-'||to_char(C.tool_data4,'99990'),' ',")||' '||C.tool_data11||' OHC',

'hook',DECODE(LEAST(C.TOOL_DATA1,C.TOOL_DATA5),NULL,'NULL','HOOK

'||REPLACE(to_char(C.tool_data1,'99990')||'-'||to_char(C.tool_data5,'90'),' ',")),

'threadmilldes',REPLACE(C.tool_data8||'-'||to_char(C.tool_data4,'99990')||'-'||to_char(C.tool_data6,'99990'),' ',")||' '||C.tool_data12,

'pitchrange',REPLACE(to_char(C.tool_data4,'99990')||'-'||to_char(C.tool_data6,'99990')||C.Tool_data12,' ',"),

'pitchrange2',REPLACE(to_char(C.tool_data4,'99990')||'-'||to_char(C.tool_data3,'99990')||C.Tool_data12,' ',"),

'mastercut','MASTERCUT',

'set_info','MATCHED CUTTER SET',

'null','NULL',

'Error'),' '))||chr(39)||',||'NULL'||');', /*end of alpha*/

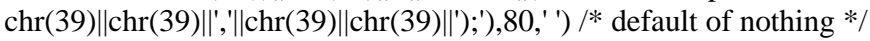

from kcp_tool_mig A, compdetail B, assembly.archive_cutting_tools C,

assembly.shank_link_codes D

where A.toolcassid is not null AND A.toolgroupid is not null

and A.toolcassid $=$ B.toolcassid

and A.toolgroupid $=$ B.toolgroupid

and A.source $=$ B.source

and A.version $=$ B.version

and A.ps_status <> 'Delete' and A.ps_status <>'EXP'

\&LIMITRESULTS

\&NOCTOOLS

\&NOSCTOOLS

and A.cutting_tool_no = C.cutting_tool_no

and A.archive_date $=$ C.archive_date

and A.source = 'archive_cutting_tools'

and C.tool_data11 $=$ D.shank_xref $(+)$

order by A.compid, B.CLASSFIELDPOS;

\section{PROMPT}

PROMPT /* Delete previous Inserts in TDM_COMP Entries */

select rpad('Delete from SCHEMA.DETAIL_TABLE where compid = '||chr(39)||A.compid||chr(39)||';',80,' ')

from kcp_tool_mig A

where A.toolcassid is not null AND A.toolgroupid is not null

and source $=$ 'inserts'

and A.ps_status <> 'Delete' and A.ps_status <>'EXP'

\&LIMITRESULTS

\&NOINSERTS

order by A.compid;

PROMPT

PROMPT

PROMPT /* Insert new Inserts SCHEMA.DETAIL_TABLE values */

select rpad('PROMPT '||A.source||', '||A.compid,80,' ')\|

rpad('Insert into SCHEMA.DETAIL_TABLE(ID,FIELDNAME,VALUE1,VALUE2) ',80,' ')\|

rpad(' values ( ' $\mid$

chr(39)||rtrim(A.compid,' ')||chr(39)||','|| /*component id*/

$\operatorname{chr}(39) \|$ |rtrim(B.CLASSFIELDPOS,' ')||chr(39)||','||/*field position*/

DECODE(B.datatype, ' $\mathrm{N}$ ' $*$ first response is for numberic values */,

chr(39)||chr(39)||','||rtrim(decode(B.sfield, /*start of numeric values */

'radius',DECODE(C.RADIUS,NULL,'NULL',C.RADIUS),

'pitch_range_hi',DECODE(C.PITCH_RANGE_HI,NULL,'NULL',C.PITCH_RANGE_HI),

'pitch_range_lo',DECODE(C.PITCH_RANGE_LO,NULL,'NULL',C.PITCH_RANGE_LO),

'width_hi',DECODE(C.WIDTH_HI,NULL,'NULL',C.WIDTH_HI), 


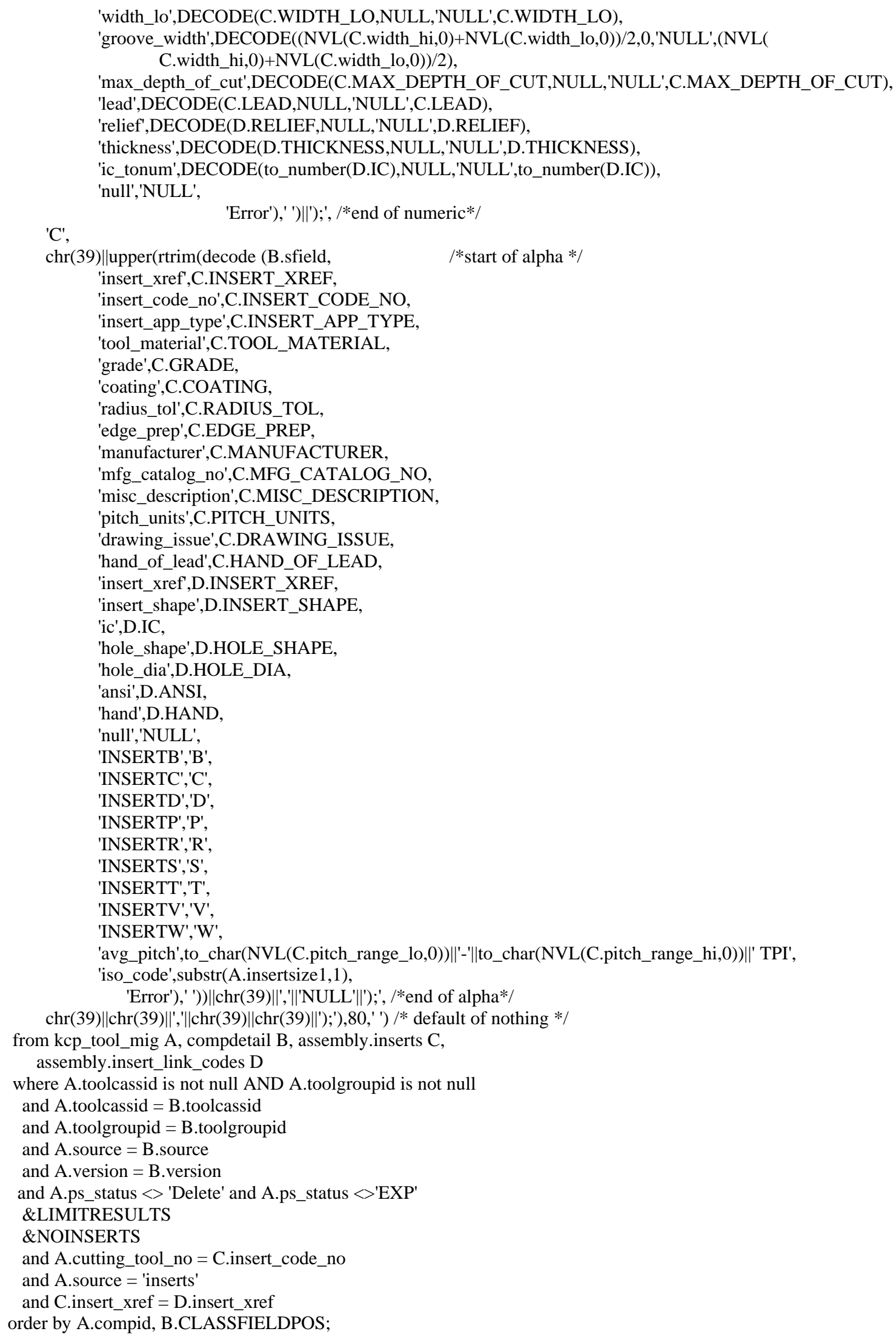




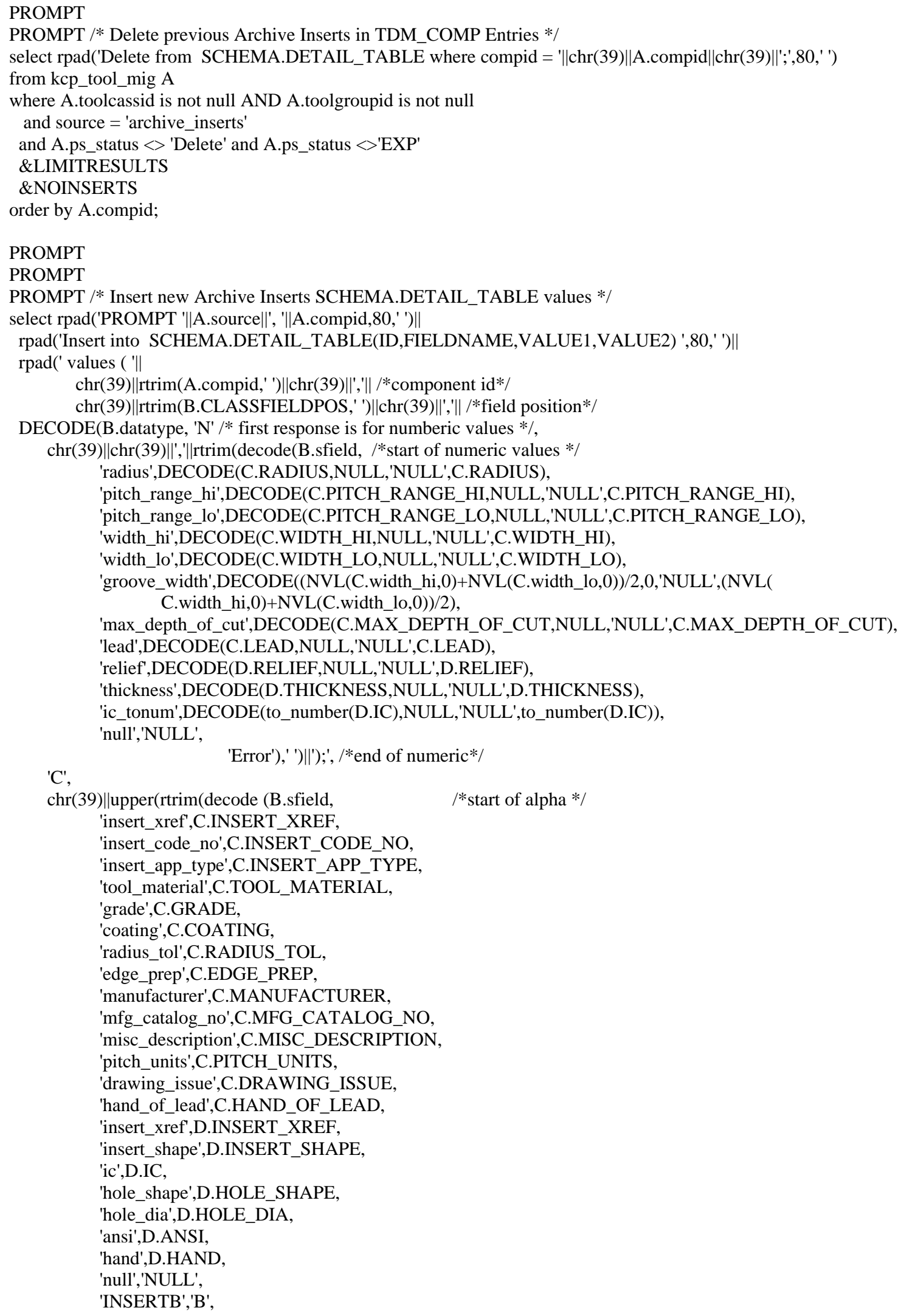




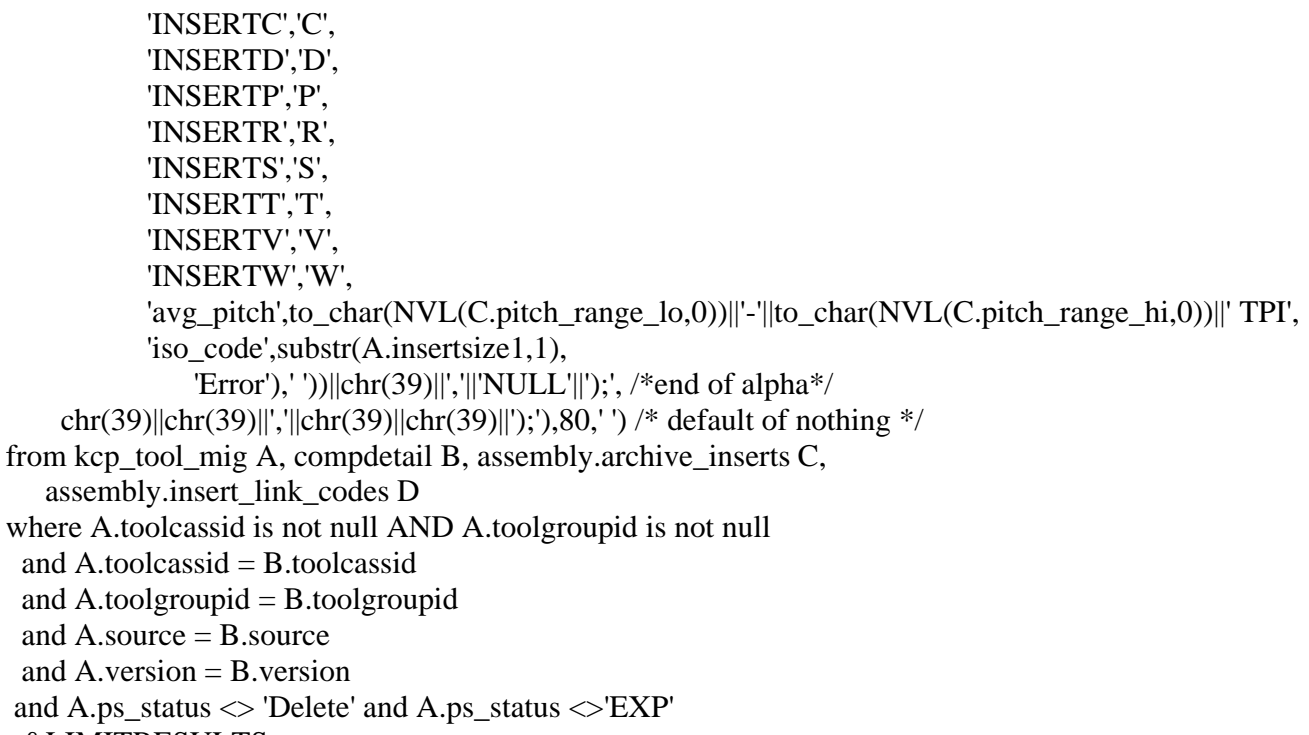

Spool off

UNDEFINE LIMITRESULTS

UNDEFINE NOCOLLETS

UNDEFINE NOHOLDERS

UNDEFINE NOEXTENSIONS

UNDEFINE NOINSERTS

UNDEFINE NOSPARES

UNDEFINE NOCTOOLS

UNDEFINE NOSCTOOLS

UNDEFINE REMSHANKS

set verify on

set showmode on

set echo on

set heading on

set Feedback 6

set tab off

set trim off

set headsep on

set embedded off

set wrap off

set line 600 


\section{Appendix C}

\section{Sample Output From the SQL Export}

set pagesize 6000

Note: Actual File was 426,380 lines

set linesize 300

REM @ X:/PROCES 1/PROJECTS/CTOOLD 1/MIGRAT 1/MIGRAT 1/Prodver/lcompdet.sql

spool X:/PROCES 1/PROJECTS/CTOOLD 1/MIGRAT 1/MIGRAT 1/Prodver/lcompdet.txt

set scan off

/* Delete previous EXTENSIONS in TDM_COMP Entries */

Delete from SCHEMA.DETAIL_TABLE where compid = 'BYHE';

Delete from SCHEMA.DETAIL_TABLE where compid = 'CKNS';

Delete from SCHEMA.DETAIL_TABLE where compid = 'CQUW';

Delete from SCHEMA.DETAIL_TABLE where compid = 'CZOQ';

Delete from SCHEMA.DETAIL_TABLE where compid = 'DNEK';

Delete from SCHEMA.DETAIL_TABLE where compid = 'DRKH';

Delete from SCHEMA.DETAIL_TABLE where compid = 'DZFT';

Delete from SCHEMA.DETAIL_TABLE where compid = 'EKPF';

Delete from SCHEMA.DETAIL_TABLE where compid = 'FDRJ';

Delete from SCHEMA.DETAIL_TABLE where compid = 'FHIB';

Delete from SCHEMA.DETAIL_TABLE where compid = 'FKTE';

Delete from SCHEMA.DETAIL_TABLE where compid = 'FPOM';

Delete from SCHEMA.DETAIL_TABLE where compid = 'FSHM';

Delete from SCHEMA.DETAIL_TABLE where compid = 'FVQQ';

Delete from SCHEMA.DETAIL_TABLE where compid = 'GJNQ';

/* Insert new Extension TDM_COMPVALUES values */

PROMPT extensions, BYHE

Insert into SCHEMA.DETAIL_TABLE (ID,FIELDNAME,VALUE1,VALUE2)

values ( 'BYHE','1',",3);

PROMPT extensions, BYHE

Insert into SCHEMA.DETAIL_TABLE (ID,FIELDNAME,VALUE1,VALUE2)

values ( 'BYHE','4',",.44);

PROMPT extensions, BYHE

Insert into SCHEMA.DETAIL_TABLE (ID,FIELDNAME,VALUE1,VALUE2)

values ( 'BYHE','5',",1.5);

PROMPT extensions, BYHE

Insert into SCHEMA.DETAIL_TABLE (ID,FIELDNAME,VALUE1,VALUE2)

values ( 'BYHE','6',",.44);

PROMPT extensions, BYHE

Insert into SCHEMA.DETAIL_TABLE (ID,FIELDNAME,VALUE1,VALUE2)

values ( 'BYHE','9',",4.5);

PROMPT extensions, BYHE

Insert into SCHEMA.DETAIL_TABLE (ID,FIELDNAME,VALUE1,VALUE2)

values ( 'BYHE','13',",0);

PROMPT extensions, BYHE

Insert into SCHEMA.DETAIL_TABLE (ID,FIELDNAME,VALUE1,VALUE2)

values ( 'BYHE','129','UNIVERSAL',NULL);

PROMPT extensions, BYHE

Insert into SCHEMA.DETAIL_TABLE (ID,FIELDNAME,VALUE1,VALUE2) 
values ( 'BYHE','130','508030',NULL);

PROMPT extensions, CKNS

Insert into SCHEMA.DETAIL_TABLE (ID,FIELDNAME,VALUE1,VALUE2)

values ( 'CKNS','1',",3.12);

PROMPT extensions, CKNS

Insert into SCHEMA.DETAIL_TABLE (ID,FIELDNAME,VALUE1,VALUE2)

values ( 'CKNS','4',",.62);

PROMPT extensions, CKNS

Insert into SCHEMA.DETAIL_TABLE (ID,FIELDNAME,VALUE1,VALUE2)

values ( 'CKNS','5',",1.5);

PROMPT extensions, CKNS

Insert into SCHEMA.DETAIL_TABLE (ID,FIELDNAME,VALUE1,VALUE2)

values ( 'CKNS','6',",.62);

PROMPT extensions, CKNS

Insert into SCHEMA.DETAIL_TABLE (ID,FIELDNAME,VALUE1,VALUE2)

values ( 'CKNS','9',",4.62);

PROMPT extensions, CKNS

Insert into SCHEMA.DETAIL_TABLE (ID,FIELDNAME,VALUE1,VALUE2)

values ( 'CKNS','13',",0);

PROMPT extensions, CKNS

Insert into SCHEMA.DETAIL_TABLE (ID,FIELDNAME,VALUE1,VALUE2)

values ( 'CKNS','129','UNIVERSAL',NULL);

PROMPT extensions, CKNS

Insert into SCHEMA.DETAIL_TABLE (ID,FIELDNAME,VALUE1,VALUE2)

values ( 'CKNS','130','508033',NULL);

PROMPT extensions, CQUW

Insert into SCHEMA.DETAIL_TABLE (ID,FIELDNAME,VALUE1,VALUE2)

values ( 'CQUW','1',",3.12);

PROMPT extensions, CQUW

Insert into SCHEMA.DETAIL_TABLE (ID,FIELDNAME,VALUE1,VALUE2)

values ( 'CQUW','4',",.62);

PROMPT extensions, CQUW

Insert into SCHEMA.DETAIL_TABLE (ID,FIELDNAME,VALUE1,VALUE2)

values ( 'CQUW','5',",1.5);

PROMPT extensions, CQUW

Insert into SCHEMA.DETAIL_TABLE (ID,FIELDNAME,VALUE1,VALUE2)

values ( 'CQUW','6',",.62);

PROMPT extensions, CQUW

Insert into SCHEMA.DETAIL_TABLE (ID,FIELDNAME,VALUE1,VALUE2)

values ( 'CQUW','9',",4.62);

PROMPT extensions, CQUW

Insert into SCHEMA.DETAIL_TABLE (ID,FIELDNAME,VALUE1,VALUE2)

values ( 'CQUW','13',",0);

PROMPT extensions, CQUW

Insert into SCHEMA.DETAIL_TABLE (ID,FIELDNAME,VALUE1,VALUE2)

values ( 'CQUW','129','UNIVERSAL',NULL); 
PROMPT extensions, CQUW

Insert into SCHEMA.DETAIL_TABLE (ID,FIELDNAME,VALUE1,VALUE2)

values ( 'CQUW','130','508036',NULL);

PROMPT extensions, CZOQ

Insert into SCHEMA.DETAIL_TABLE (ID,FIELDNAME,VALUE1,VALUE2)

values ( 'CZOQ','1',",3.5);

PROMPT extensions, CZOQ

Insert into SCHEMA.DETAIL_TABLE (ID,FIELDNAME,VALUE1,VALUE2)

values ( 'CZOQ','2',",5.4);

PROMPT extensions, CZOQ

Insert into SCHEMA.DETAIL_TABLE (ID,FIELDNAME,VALUE1,VALUE2)

values ( 'CZOQ','5',",1.5);

PROMPT extensions, CZOQ

Insert into SCHEMA.DETAIL_TABLE (ID,FIELDNAME,VALUE1,VALUE2)

values ( 'CZOQ','6',",3.5);

PROMPT extensions, CZOQ

Insert into SCHEMA.DETAIL_TABLE (ID,FIELDNAME,VALUE1,VALUE2)

values ( 'CZOQ','7',",2.626);

PROMPT extensions, CZOQ

Insert into SCHEMA.DETAIL_TABLE (ID,FIELDNAME,VALUE1,VALUE2)

values ( 'CZOQ','11',",.375);

PROMPT extensions, CZOQ

Insert into SCHEMA.DETAIL_TABLE (ID,FIELDNAME,VALUE1,VALUE2)

values ( 'CZOQ','13',",1.003);

PROMPT extensions, CZOQ

Insert into SCHEMA.DETAIL_TABLE (ID,FIELDNAME,VALUE1,VALUE2)

values ( 'CZOQ','14',",0);

PROMPT extensions, CZOQ

Insert into SCHEMA.DETAIL_TABLE (ID,FIELDNAME,VALUE1,VALUE2)

values ( 'CZOQ','129','CRITERION',NULL);

PROMPT extensions, CZOQ

Insert into SCHEMA.DETAIL_TABLE (ID,FIELDNAME,VALUE1,VALUE2)

values ( 'CZOQ','130','DBL-152',NULL);

PROMPT extensions, DNEK

Insert into SCHEMA.DETAIL_TABLE (ID,FIELDNAME,VALUE1,VALUE2)

values ( 'DNEK','1',",5);

PROMPT extensions, DNEK

Insert into SCHEMA.DETAIL_TABLE (ID,FIELDNAME,VALUE1,VALUE2)

values ( 'DNEK','2',",7.6);

PROMPT extensions, DNEK

Insert into SCHEMA.DETAIL_TABLE (ID,FIELDNAME,VALUE1,VALUE2)

values ( 'DNEK','5',",2);

PROMPT extensions, DNEK

Insert into SCHEMA.DETAIL_TABLE (ID,FIELDNAME,VALUE1,VALUE2)

values ( 'DNEK','6',",5);

PROMPT extensions, DNEK

Insert into SCHEMA.DETAIL_TABLE (ID,FIELDNAME,VALUE1,VALUE2) 
values ( 'DNEK','7',",2);

PROMPT extensions, DNEK

Insert into SCHEMA.DETAIL_TABLE (ID,FIELDNAME,VALUE1,VALUE2)

values ( 'DNEK','11',",.5);

PROMPT extensions, DNEK

Insert into SCHEMA.DETAIL_TABLE (ID,FIELDNAME,VALUE1,VALUE2)

values ( 'DNEK','13',",1.25);

PROMPT extensions, DNEK

Insert into SCHEMA.DETAIL_TABLE (ID,FIELDNAME,VALUE1,VALUE2)

values ( 'DNEK','14',",0);

PROMPT extensions, DNEK

Insert into SCHEMA.DETAIL_TABLE (ID,FIELDNAME,VALUE1,VALUE2)

values ( 'DNEK','129','CRITERION',NULL);

PROMPT extensions, DNEK

Insert into SCHEMA.DETAIL_TABLE (ID,FIELDNAME,VALUE1,VALUE2)

values ( 'DNEK','130','DBL-202',NULL);

PROMPT extensions, DRKH

Insert into SCHEMA.DETAIL_TABLE (ID,FIELDNAME,VALUE1,VALUE2)

values ( 'DRKH','1',",6.5);

PROMPT extensions, DRKH

Insert into SCHEMA.DETAIL_TABLE (ID,FIELDNAME,VALUE1,VALUE2)

values ( 'DRKH','2',",8.9);

PROMPT extensions, DRKH

Insert into SCHEMA.DETAIL_TABLE (ID,FIELDNAME,VALUE1,VALUE2)

values ( 'DRKH','5',",2.5);

PROMPT extensions, DRKH

Insert into SCHEMA.DETAIL_TABLE (ID,FIELDNAME,VALUE1,VALUE2)

values ( 'DRKH','6',",6.5);

PROMPT extensions, DRKH

Insert into SCHEMA.DETAIL_TABLE (ID,FIELDNAME,VALUE1,VALUE2)

values ( 'DRKH','7',",2.5);

PROMPT extensions, DRKH

Insert into SCHEMA.DETAIL_TABLE (ID,FIELDNAME,VALUE1,VALUE2)

values ( 'DRKH','11',",.5);

PROMPT extensions, DRKH

Insert into SCHEMA.DETAIL_TABLE (ID,FIELDNAME,VALUE1,VALUE2)

values ( 'DRKH','13',",1.25);

PROMPT extensions, DRKH

Insert into SCHEMA.DETAIL_TABLE (ID,FIELDNAME,VALUE1,VALUE2)

values ( 'DRKH','14',",0);

PROMPT extensions, DRKH

Insert into SCHEMA.DETAIL_TABLE (ID,FIELDNAME,VALUE1,VALUE2)

values ( 'DRKH','129','CRITERION',NULL);

PROMPT extensions, DRKH

Insert into SCHEMA.DETAIL_TABLE (ID,FIELDNAME,VALUE1,VALUE2)

values ( 'DRKH','130','TS-250',NULL); 
PROMPT extensions, DZFT

Insert into SCHEMA.DETAIL_TABLE (ID,FIELDNAME,VALUE1,VALUE2)

values ( 'DZFT','1',",5.75);

PROMPT extensions, DZFT

Insert into SCHEMA.DETAIL_TABLE (ID,FIELDNAME,VALUE1,VALUE2)

values ( 'DZFT','2',",9.15);

PROMPT extensions, DZFT

Insert into SCHEMA.DETAIL_TABLE (ID,FIELDNAME,VALUE1,VALUE2)

values ( 'DZFT','5',",2.75);

PROMPT extensions, DZFT

Insert into SCHEMA.DETAIL_TABLE (ID,FIELDNAME,VALUE1,VALUE2)

values ( 'DZFT','6',",5.75);

PROMPT extensions, DZFT

Insert into SCHEMA.DETAIL_TABLE (ID,FIELDNAME,VALUE1,VALUE2)

values ( 'DZFT','7',",2.75);

PROMPT extensions, DZFT

Insert into SCHEMA.DETAIL_TABLE (ID,FIELDNAME,VALUE1,VALUE2)

values ( 'DZFT','11',",.5);

PROMPT extensions, DZFT

Insert into SCHEMA.DETAIL_TABLE (ID,FIELDNAME,VALUE1,VALUE2)

values ( 'DZFT','13',",0);

PROMPT extensions, DZFT

Insert into SCHEMA.DETAIL_TABLE (ID,FIELDNAME,VALUE1,VALUE2)

values ( 'DZFT','14',",0);

PROMPT extensions, DZFT

Insert into SCHEMA.DETAIL_TABLE (ID,FIELDNAME,VALUE1,VALUE2)

values ( 'DZFT','129','CRITERION',NULL);

PROMPT extensions, DZFT

Insert into SCHEMA.DETAIL_TABLE (ID,FIELDNAME,VALUE1,VALUE2)

values ( 'DZFT','130','S-2',NULL);

PROMPT extensions, EKPF

Insert into SCHEMA.DETAIL_TABLE (ID,FIELDNAME,VALUE1,VALUE2)

values ( 'EKPF','1',",5.75);

PROMPT extensions, EKPF

Insert into SCHEMA.DETAIL_TABLE (ID,FIELDNAME,VALUE1,VALUE2)

values ( 'EKPF','2',",9.15);

PROMPT extensions, EKPF

Insert into SCHEMA.DETAIL_TABLE (ID,FIELDNAME,VALUE1,VALUE2)

values ( 'EKPF','5',",2.75);

PROMPT extensions, EKPF

Insert into SCHEMA.DETAIL_TABLE (ID,FIELDNAME,VALUE1,VALUE2)

values ( 'EKPF','6',",5.75);

PROMPT extensions, EKPF

Insert into SCHEMA.DETAIL_TABLE (ID,FIELDNAME,VALUE1,VALUE2)

values ( 'EKPF','7',",2.75);

PROMPT extensions, EKPF

Insert into SCHEMA.DETAIL_TABLE (ID,FIELDNAME,VALUE1,VALUE2) 
values ( 'EKPF','11',",.375);

PROMPT extensions, EKPF

Insert into SCHEMA.DETAIL_TABLE (ID,FIELDNAME,VALUE1,VALUE2)

values ( 'EKPF','13',",0);

PROMPT extensions, EKPF

Insert into SCHEMA.DETAIL_TABLE (ID,FIELDNAME,VALUE1,VALUE2)

values ( 'EKPF','14',",0);

PROMPT extensions, EKPF

Insert into SCHEMA.DETAIL_TABLE (ID,FIELDNAME,VALUE1,VALUE2)

values ( 'EKPF','129','CRITERION',NULL);

PROMPT extensions, EKPF

Insert into SCHEMA.DETAIL_TABLE (ID,FIELDNAME,VALUE1,VALUE2)

values ( 'EKPF','130','S-2A',NULL);

PROMPT extensions, FDRJ

Insert into SCHEMA.DETAIL_TABLE (ID,FIELDNAME,VALUE1,VALUE2)

values ( 'FDRJ','1',",4);

PROMPT extensions, FDRJ

Insert into SCHEMA.DETAIL_TABLE (ID,FIELDNAME,VALUE1,VALUE2)

values ( 'FDRJ','4',",.625);

PROMPT extensions, FDRJ

Insert into SCHEMA.DETAIL_TABLE (ID,FIELDNAME,VALUE1,VALUE2)

values ( 'FDRJ','5',",1.28);

PROMPT extensions, FDRJ

Insert into SCHEMA.DETAIL_TABLE (ID,FIELDNAME,VALUE1,VALUE2)

values ( 'FDRJ','6',",.63);

PROMPT extensions, FDRJ

Insert into SCHEMA.DETAIL_TABLE (ID,FIELDNAME,VALUE1,VALUE2)

values ( 'FDRJ','8',",NULL);

PROMPT extensions, FDRJ

Insert into SCHEMA.DETAIL_TABLE (ID,FIELDNAME,VALUE1,VALUE2)

values ( 'FDRJ','9',",6.72);

PROMPT extensions, FDRJ

Insert into SCHEMA.DETAIL_TABLE (ID,FIELDNAME,VALUE1,VALUE2)

values ( 'FDRJ','129','DEVLIEG',NULL);

PROMPT extensions, FDRJ

Insert into SCHEMA.DETAIL_TABLE (ID,FIELDNAME,VALUE1,VALUE2)

values ( 'FDRJ','130','511-878 (MODIFIED)',NULL);

PROMPT extensions, FHIB

Insert into SCHEMA.DETAIL_TABLE (ID,FIELDNAME,VALUE1,VALUE2)

values ( 'FHIB','1',",4.25);

PROMPT extensions, FHIB

Insert into SCHEMA.DETAIL_TABLE (ID,FIELDNAME,VALUE1,VALUE2)

values ( 'FHIB','4',",1.45);

PROMPT extensions, FHIB

Insert into SCHEMA.DETAIL_TABLE (ID,FIELDNAME,VALUE1,VALUE2)

values ( 'FHIB','5',",4.25); 
PROMPT extensions, FHIB

Insert into SCHEMA.DETAIL_TABLE (ID,FIELDNAME,VALUE1,VALUE2)

values ( 'FHIB','6',",1.45);

PROMPT extensions, FHIB

Insert into SCHEMA.DETAIL_TABLE (ID,FIELDNAME,VALUE1,VALUE2)

values ( 'FHIB','9',",6.75);

PROMPT extensions, FHIB

Insert into SCHEMA.DETAIL_TABLE (ID,FIELDNAME,VALUE1,VALUE2)

values ( 'FHIB','13',",.75);

PROMPT extensions, FHIB

Insert into SCHEMA.DETAIL_TABLE (ID,FIELDNAME,VALUE1,VALUE2)

values ( 'FHIB','129','PARLEC',NULL);

PROMPT extensions, FHIB

Insert into SCHEMA.DETAIL_TABLE (ID,FIELDNAME,VALUE1,VALUE2)

values ( 'FHIB','130','S10-10TA4',NULL);

PROMPT extensions, FKTE

Insert into SCHEMA.DETAIL_TABLE (ID,FIELDNAME,VALUE1,VALUE2)

values ( 'FKTE','1',",5);

PROMPT extensions, FKTE

Insert into SCHEMA.DETAIL_TABLE (ID,FIELDNAME,VALUE1,VALUE2)

values ( 'FKTE','2',",7.6);

PROMPT extensions, FKTE

Insert into SCHEMA.DETAIL_TABLE (ID,FIELDNAME,VALUE1,VALUE2)

values ( 'FKTE','5',",2);

PROMPT extensions, FKTE

Insert into SCHEMA.DETAIL_TABLE (ID,FIELDNAME,VALUE1,VALUE2)

values ( 'FKTE','6',",5);

PROMPT extensions, FKTE

Insert into SCHEMA.DETAIL_TABLE (ID,FIELDNAME,VALUE1,VALUE2)

values ( 'FKTE','7',",2);

PROMPT extensions, FKTE

Insert into SCHEMA.DETAIL_TABLE (ID,FIELDNAME,VALUE1,VALUE2)

values ( 'FKTE','11',",.375);

PROMPT extensions, FKTE

Insert into SCHEMA.DETAIL_TABLE (ID,FIELDNAME,VALUE1,VALUE2)

values ( 'FKTE','13',",1.25);

PROMPT extensions, FKTE

Insert into SCHEMA.DETAIL_TABLE (ID,FIELDNAME,VALUE1,VALUE2)

values ( 'FKTE','14',",0);

PROMPT extensions, FKTE

Insert into SCHEMA.DETAIL_TABLE (ID,FIELDNAME,VALUE1,VALUE2)

values ( 'FKTE','129','CRITERION',NULL);

PROMPT extensions, FKTE

Insert into SCHEMA.DETAIL_TABLE (ID,FIELDNAME,VALUE1,VALUE2)

values ( 'FKTE','130','DBL-202A',NULL);

PROMPT extensions, FPOM

Insert into SCHEMA.DETAIL_TABLE (ID,FIELDNAME,VALUE1,VALUE2) 
values ( 'FPOM','1',",3.5);

PROMPT extensions, FPOM

Insert into SCHEMA.DETAIL_TABLE (ID,FIELDNAME,VALUE1,VALUE2)

values ( 'FPOM','2',",5.4);

PROMPT extensions, FPOM

Insert into SCHEMA.DETAIL_TABLE (ID,FIELDNAME,VALUE1,VALUE2)

values ( 'FPOM','5',",1.5);

PROMPT extensions, FPOM

Insert into SCHEMA.DETAIL_TABLE (ID,FIELDNAME,VALUE1,VALUE2)

values ( 'FPOM','6',",3.5);

PROMPT extensions, FPOM

Insert into SCHEMA.DETAIL_TABLE (ID,FIELDNAME,VALUE1,VALUE2)

values ( 'FPOM','7',",2.626);

PROMPT extensions, FPOM

Insert into SCHEMA.DETAIL_TABLE (ID,FIELDNAME,VALUE1,VALUE2)

values ( 'FPOM','11',",.375);

PROMPT extensions, FPOM

Insert into SCHEMA.DETAIL_TABLE (ID,FIELDNAME,VALUE1,VALUE2)

values ( 'FPOM','13',",1.003);

PROMPT extensions, FPOM

Insert into SCHEMA.DETAIL_TABLE (ID,FIELDNAME,VALUE1,VALUE2)

values ( 'FPOM','14',",0);

PROMPT extensions, FPOM

Insert into SCHEMA.DETAIL_TABLE (ID,FIELDNAME,VALUE1,VALUE2)

values ( 'FPOM','129','CRITERION',NULL);

PROMPT extensions, FPOM

Insert into SCHEMA.DETAIL_TABLE (ID,FIELDNAME,VALUE1,VALUE2)

values ( 'FPOM','130','DBL-152A',NULL);

PROMPT extensions, FSHM

Insert into SCHEMA.DETAIL_TABLE (ID,FIELDNAME,VALUE1,VALUE2)

values ( 'FSHM','1',",3.8);

PROMPT extensions, FSHM

Insert into SCHEMA.DETAIL_TABLE (ID,FIELDNAME,VALUE1,VALUE2)

values ( 'FSHM','2',",5.7);

PROMPT extensions, FSHM

Insert into SCHEMA.DETAIL_TABLE (ID,FIELDNAME,VALUE1,VALUE2)

values ( 'FSHM','5',",1.5);

PROMPT extensions, FSHM

Insert into SCHEMA.DETAIL_TABLE (ID,FIELDNAME,VALUE1,VALUE2)

values ( 'FSHM','6',",3.8);

PROMPT extensions, FSHM

Insert into SCHEMA.DETAIL_TABLE (ID,FIELDNAME,VALUE1,VALUE2)

values ( 'FSHM','7',",2.626);

PROMPT extensions, FSHM

Insert into SCHEMA.DETAIL_TABLE (ID,FIELDNAME,VALUE1,VALUE2)

values ( 'FSHM','11',",.5); 
PROMPT extensions, FSHM

Insert into SCHEMA.DETAIL_TABLE (ID,FIELDNAME,VALUE1,VALUE2)

values ( 'FSHM','13',",0);

PROMPT extensions, FSHM

Insert into SCHEMA.DETAIL_TABLE (ID,FIELDNAME,VALUE1,VALUE2)

values ( 'FSHM','14',",0);

PROMPT extensions, FSHM

Insert into SCHEMA.DETAIL_TABLE (ID,FIELDNAME,VALUE1,VALUE2)

values ( 'FSHM','129','CRITERION',NULL);

PROMPT extensions, FSHM

Insert into SCHEMA.DETAIL_TABLE (ID,FIELDNAME,VALUE1,VALUE2)

values ( 'FSHM','130','002A',NULL);

PROMPT extensions, FVQQ

Insert into SCHEMA.DETAIL_TABLE (ID,FIELDNAME,VALUE1,VALUE2)

values ( 'FVQQ','1',",3.8);

PROMPT extensions, FVQQ

Insert into SCHEMA.DETAIL_TABLE (ID,FIELDNAME,VALUE1,VALUE2)

values ( 'FVQQ','2',",5.7);

PROMPT extensions, FVQQ

Insert into SCHEMA.DETAIL_TABLE (ID,FIELDNAME,VALUE1,VALUE2)

values ( 'FVQQ','5',",1.5);

PROMPT extensions, FVQQ

Insert into SCHEMA.DETAIL_TABLE (ID,FIELDNAME,VALUE1,VALUE2)

values ( 'FVQQ','6',",3.8);

PROMPT extensions, FVQQ

Insert into SCHEMA.DETAIL_TABLE (ID,FIELDNAME,VALUE1,VALUE2)

values ( 'FVQQ','7',",2.626);

PROMPT extensions, FVQQ

Insert into SCHEMA.DETAIL_TABLE (ID,FIELDNAME,VALUE1,VALUE2)

values ( 'FVQQ','11',",.5);

PROMPT extensions, FVQQ

Insert into SCHEMA.DETAIL_TABLE (ID,FIELDNAME,VALUE1,VALUE2)

values ( 'FVQQ','13',",0);

PROMPT extensions, FVQQ

Insert into SCHEMA.DETAIL_TABLE (ID,FIELDNAME,VALUE1,VALUE2)

values ( 'FVQQ','14',",0);

PROMPT extensions, FVQQ

Insert into SCHEMA.DETAIL_TABLE (ID,FIELDNAME,VALUE1,VALUE2)

values ( 'FVQQ','129','CRITERION',NULL);

PROMPT extensions, FVQQ

Insert into SCHEMA.DETAIL_TABLE (ID,FIELDNAME,VALUE1,VALUE2)

values ( 'FVQQ','130','002B',NULL);

PROMPT extensions, GJNQ

Insert into SCHEMA.DETAIL_TABLE (ID,FIELDNAME,VALUE1,VALUE2)

values ( 'GJNQ','1',",3.62);

PROMPT extensions, GJNQ

Insert into SCHEMA.DETAIL_TABLE (ID,FIELDNAME,VALUE1,VALUE2) 
values ( 'GJNQ','4',",1.9);

PROMPT extensions, GJNQ

Insert into SCHEMA.DETAIL_TABLE (ID,FIELDNAME,VALUE1,VALUE2) values ( 'GJNQ','5',",1.875);

PROMPT extensions, GJNQ

Insert into SCHEMA.DETAIL_TABLE (ID,FIELDNAME,VALUE1,VALUE2)

values ( 'GJNQ','6',",2.5);

PROMPT extensions, GJNQ

Insert into SCHEMA.DETAIL_TABLE (ID,FIELDNAME,VALUE1,VALUE2)

values ( 'GJNQ','8',",NULL);

PROMPT extensions, GJNQ

Insert into SCHEMA.DETAIL_TABLE (ID,FIELDNAME,VALUE1,VALUE2)

values ( 'GJNQ','9',",7.12);

PROMPT extensions, GJNQ

Insert into SCHEMA.DETAIL_TABLE (ID,FIELDNAME,VALUE1,VALUE2)

values ( 'GJNQ','129','UNIVERSAL',NULL);

PROMPT extensions, GJNQ

Insert into SCHEMA.DETAIL_TABLE (ID,FIELDNAME,VALUE1,VALUE2)

values ( 'GJNQ','130','AF241755',NULL);

commit;

set scan off

spool off 